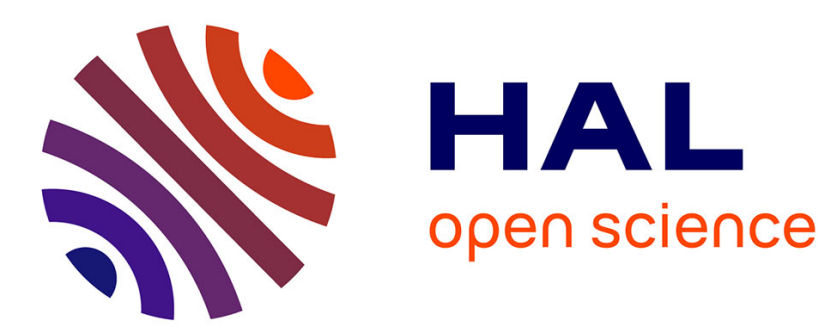

\title{
Effective Detection of Sleep-in-Atomic-Context Bugs in the Linux Kernel
}

\author{
Jia-Ju Bai, Julia Lawall, Shi-Min Hu
}

\section{To cite this version:}

Jia-Ju Bai, Julia Lawall, Shi-Min Hu. Effective Detection of Sleep-in-Atomic-Context Bugs in the Linux Kernel. ACM Transactions on Computer Systems, 2020, 36 (4), pp.10. 10.1145/3381990 . hal-03032244

\section{HAL Id: hal-03032244 \\ https://hal.inria.fr/hal-03032244}

Submitted on 30 Nov 2020

HAL is a multi-disciplinary open access archive for the deposit and dissemination of scientific research documents, whether they are published or not. The documents may come from teaching and research institutions in France or abroad, or from public or private research centers.
L'archive ouverte pluridisciplinaire HAL, est destinée au dépôt et à la diffusion de documents scientifiques de niveau recherche, publiés ou non, émanant des établissements d'enseignement et de recherche français ou étrangers, des laboratoires publics ou privés. 


\title{
Effective Detection of Sleep-in-Atomic-Context Bugs in the Linux Kernel*
}

\author{
JIA-JU BAI, Tsinghua University, China \\ JULIA LAWALL, Sorbonne University/Inria/LIP6, France \\ SHI-MIN HU, Tsinghua University, China
}

Atomic context is an execution state of the Linux kernel, in which kernel code monopolizes a CPU core. In this state, the Linux kernel may only perform operations that cannot sleep, as otherwise a system hang or crash may occur. We refer to this kind of concurrency bug as a sleep-in-atomic-context (SAC) bug. In practice, SAC bugs are hard to find, as they do not cause problems in all executions.

In this paper, we propose a practical static approach named DSAC, to effectively detect SAC bugs in the Linux kernel. DSAC uses three key techniques: (1) a summary-based analysis to identify the code that may be executed in atomic context, (2) a connection-based alias analysis to identify the set of functions referenced by a function pointer, and (3) a path-check method to filter out repeated reports and false bugs. We evaluate DSAC on Linux 4.17, and find 1159 SAC bugs. We manually check all the bugs, and find that 1068 bugs are real. We have randomly selected 300 of the real bugs and sent them to kernel developers. 220 of these bugs have been confirmed, and 51 of our patches fixing 115 bugs have been applied.

CCS Concepts: • Software and its engineering $\rightarrow$ Software defect analysis; Operating systems; • Computer systems organization $\rightarrow$ Reliability;

Additional Key Words and Phrases: Static analysis, bug detection, atomic context, operating system

\section{ACM Reference Format:}

Jia-Ju Bai, Julia Lawall, and Shi-Min Hu. 2020. Effective Detection of Sleep-in-Atomic-Context Bugs in the Linux Kernel. ACM Trans. Comput. Syst. 36, 4, Article 10 (April 2020), 30 pages. https://doi.org/0000001.0000001

\section{INTRODUCTION}

Concurrency bugs are known to be difficult to detect in the Linux kernel, because most of them are hard to reproduce in real execution. Many tools have been proposed to detect common concurrency bugs, such as atomicity violations and data races. However, as another kind of concurrency bug, sleep-in-atomic-context (SAC) bugs have received less attention. SAC bugs occur at the kernel level when a sleeping operation is performed in atomic context [18], such as when holding a spinlock or executing an interrupt handler. Code executing in atomic context monopolizes a CPU core, and the progress of other threads that need to concurrently access the same resources is delayed. Thus, the code executing in atomic context should complete as quickly as possible. Sleeping in atomic context is forbidden, because it can block a CPU core for a long time and may lead to a system hang.

\footnotetext{
*This paper extends a previous conference paper [7] in the 2018 USENIX Annual Technical Conference. This paper includes a new summary-based analysis to identify atomic context, a new connection-based alias analysis to handle function-pointer calls, and an improved path-check method to filter out repeated reports and false bugs. Compared to that work, this paper analyzes the whole kernel instead of only kernel modules, and handles function-pointer calls. This paper also finds more real bugs in a more recent Linux kernel version.
}

Authors' addresses: Jia-Ju Bai, Tsinghua University, Beijing, 100084, China, baijiaju1990@gmail.com; Julia Lawall, Sorbonne University/Inria/LIP6, Paris, France, julia.lawall@lip6.fr; Shi-Min Hu, Tsinghua University, Beijing, 100084, China, shimin@ tsinghua.edu.cn.

(C) 2020 Association for Computing Machinery.

This is the author's version of the work. It is posted here for your personal use. Not for redistribution. The definitive Version of Record was published in ACM Transactions on Computer Systems, https://doi.org/0000001.0000001. 
Even though sleeping in atomic context is forbidden, many SAC bugs still exist in the Linux kernel. The main reasons why SAC bugs continue to occur include: (1) Determining whether an operation can sleep often requires system-specific experience; (2) Some parts of the kernel code can be difficult to test, for example, testing a device driver requires its associated device; (3) SAC bugs do not always cause problems in real execution, and they are often hard to reproduce at runtime. Recent studies $[19,60]$ have shown that SAC bugs have caused serious system hangs at runtime. Thus, it is necessary to design approaches to detect them.

Many existing approaches $[13,26,37,55]$ can detect concurrency bugs, but most of them are designed for user-level applications. Some approaches [20, 25, 27, 54, 57] can detect some common kinds of kernel-level concurrency bugs, such as atomicity violations and data races, but they have not addressed SAC bugs. Several approaches $[4,15,24,46]$ can detect common kinds of OS kernel faults, including SAC bugs. But they are not specific to SAC bugs, and most of them $[15,24,46]$ are designed to collect statistics rather than report specific bugs to the user, making issues such as detection time and false positive rate less important. Besides, these approaches have not considered function pointers, so they may miss SAC bugs that involve function-pointer calls.

In this paper, we propose a static approach named DSAC ${ }^{1}$ that targets accurately and efficiently detecting SAC bugs in the Linux kernel. Specifically, DSAC consists of four phases. Firstly, DSAC compiles the source code of the Linux kernel and records the link information. Secondly, DSAC traverses the Linux kernel code to collect some useful information, such as the locations, callers and callees of direct function calls. Thirdly, using the collected information, for each source file, DSAC identifies the source files to which it is connected according to link information and direct function calls. This connection information is used to guide function-pointer analysis in the next phase. Fourthly, DSAC uses a summary-based analysis to identify the code that may be executed in atomic context. To accurately cover as much code as possible, this analysis is inter-procedural, context-sensitive and flow-sensitive. To handle function-pointer calls and find deep SAC bugs, DSAC uses a connection-based alias analysis to identify the set of functions referenced by the function pointer. In this phase, DSAC also uses a path-check method to filter out repeated reports and false bugs. We have implemented DSAC using LLVM [39]. DSAC works automatically with the given Linux kernel source code. Moreover, it can work in parallel to speed up the analysis.

Overall, we make the following contributions:

- We first analyze the main challenges of detecting SAC bugs in the Linux kernel, and then propose three key techniques to address these challenges: (1) a summary-based analysis to identify the code that may be executed in atomic context; (2) a connection-based alias analysis to identify the set of functions referenced by a function pointer; (3) a path-check method to filter out repeated reports and false bugs.

- Based on the three techniques, we propose a practical approach named DSAC, to effectively and automatically detect SAC bugs in the Linux kernel.

- We evaluate DSAC on a Linux stable version 3.17.2 and a mainline release 4.17, and find 891 and 1159 bugs respectively. We manually check these bugs, and find that 805 and 1068 are real, respectively, in these versions, giving false positive rates of $9.7 \%$ and $7.8 \% .304$ bugs real bugs in Linux 3.17.2 have been fixed in 4.17. We have randomly selected 300 of the real bugs in Linux 4.17, and sent them to kernel developers. 220 of these bugs have been confirmed, and 51 of our patches fixing 115 bugs have been applied.

The remainder of this paper is organized as follows. Section 2 presents the background. Section 3 introduces the main challenges in detecting SAC bugs. Section 4 introduces our key techniques to address these challenges. Section 5 introduces DSAC in detail. Section 6 presents the evaluation.

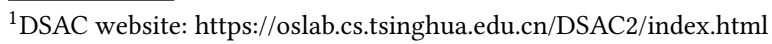




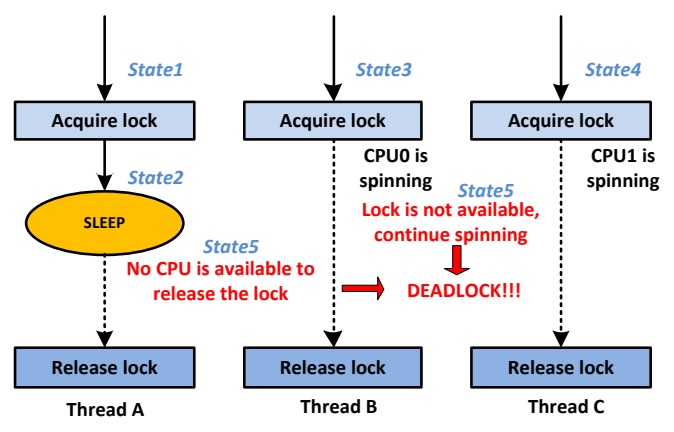

(a) Sleeping while holding a spinlock

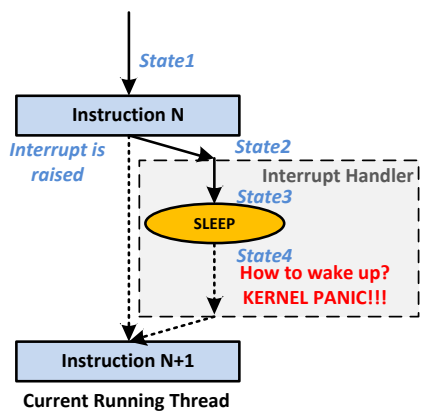

(b) Sleeping in an interrupt handler

Fig. 1. Side effects caused by sleeping in atomic context.

Section 7 compares DSAC to previous approaches. Section 8 discusses some possible extensions for DSAC. Section 9 gives the related work and Section 10 concludes the paper.

\section{BACKGROUND}

In this section, we first introduce atomic context, and then motivate our work by a real SAC bug in the Linux kernel.

\subsection{Atomic Context}

Atomic context is an OS kernel state in which a CPU core is monopolized to execute code, and the progress of other threads that need to concurrently access the same resources is delayed. This context is used to protect resources from concurrent access. In the Linux kernel, there are two common examples of atomic context, namely holding a spinlock and executing an interrupt handler. In atomic context, code execution should complete as quickly as possible without being able to be rescheduled. Due to this special situation, sleeping in atomic context is not allowed, because it can block CPU cores for long periods and may lead to a system hang or crash.

In Figure 1, we use the two common instances of atomic context to explain the side effects of sleeping in atomic context:

Sleeping while holding a spinlock. Suppose that a thread $A$ sleeps while holding a spinlock. If another thread $B$ requests the same spinlock at that time, thread $B$ will monopolize a CPU to spin until the spinlock is released by thread $A$. If all CPUs are monopolized to spin like thread $B$, there will be no CPU on which to wake up thread $A$ to release the spinlock, and thus a deadlock will occur, causing a system hang. In the single-core system, sleeping while holding a spinlock should deterministically cause a deadlock at runtime, because only one CPU is available, and it has been monopolized by thread $B$. In a multi-core system, sleeping while holding a spinlock does not always cause a deadlock at runtime, because another CPU may be available for thread $A$ on which it can release the spinlock. Figure 1(a) shows an example of the deadlock caused by sleeping while holding a spinlock. The system has two CPUs and runs from State1 to State5. The deadlock finally occurs in State5.

Sleeping in an interrupt handler. To quickly respond to interrupts, when an interrupt is raised, the Linux kernel executes the interrupt handler in the context of the currently running thread. Thus, the interrupt handler is not associated with a fixed process or thread context. For this reason, if the interrupt handler sleeps during execution, the OS scheduler cannot reschedule it because it does not have a backing process or thread, implying that it is not a schedulable entity. In this situation, a kernel panic will occur, causing a system crash. Figure 1(b) shows how a kernel panic is 


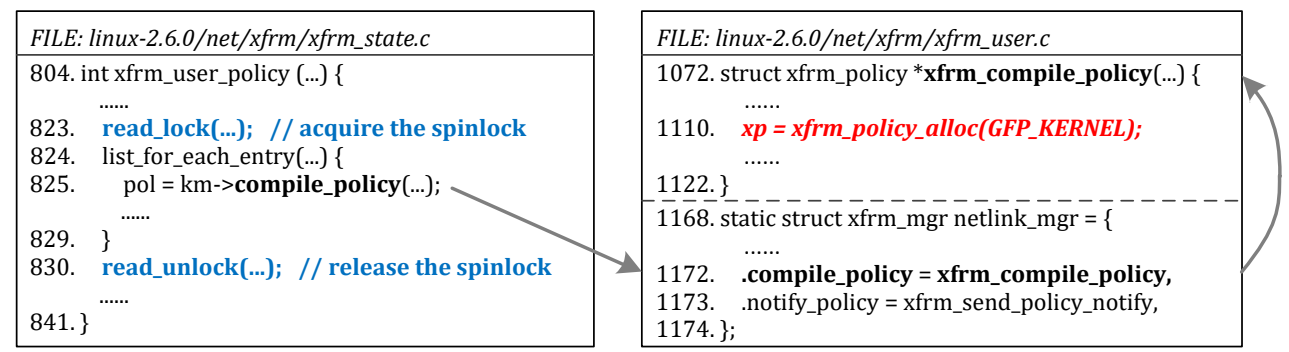

Fig. 2. Example SAC bug in the xfrm network module of Linux 2.6.0.

caused by sleeping in an interrupt handler. The system runs from State1 to State4, and a kernel panic finally occurs in State 4.

Note that atomic context only occurs at the kernel level, because user-level applications are regularly interrupted by the OS scheduler when their time slice ends. Though kernel developers often know that sleeping is not allowed in atomic context, many SAC bugs still exist in the Linux kernel $[24,46]$.

\subsection{Motivating Example}

Figure 2 shows a reported SAC bug in the Linux $x$ frm network module. This bug was introduced when the module was integrated in Linux 2.6.0 (released in December 2003), and was fixed in Linux 2.6.36 (released in October 2010), nearly 7 years later. The function xfrm_user_policy calls read_lock to acquire a spinlock on line 823 , and then calls a function pointer $\mathrm{km}->$ compile_policy

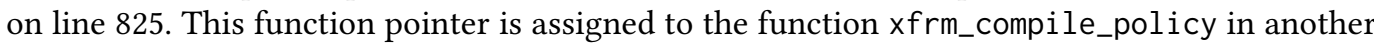
source file. The function $x f r m \_c o m p i l e \_p o l i c y$ calls $x f r m \_p o l i c y \_a l l o c$ with GFP_KERNEL to allocate a policy-related data structure on line 1110. According to the Linux kernel documentation [1], xfrm_policy_alloc called with GFP_KERNEL can sleep at runtime, thus this code contains a SAC bug. To fix this bug, the commit 2f09a4d5daaa ${ }^{2}$ replaced GFP_KERNEL with GFP_ATOMIC, which prevents the allocation from sleeping.

This example illustrates four main reasons why SAC bugs occur in the Linux kernel. (1) Determining whether an operation can sleep requires OS-specific knowledge. In this example, without experience in Linux kernel development, it may be hard to know that the function xfrm_policy_alloc called with GFP_KERNEL can sleep at runtime. (2) SAC bugs do not always cause problems in real execution and are hard to reproduce at runtime. In this example, the function $\mathrm{xfrm}$ _policy_alloc called with GFP_KERNEL only sleeps when memory is insufficient. Even in a low-memory situation, this SAC bug is not always triggered at runtime in a multi-core system, because of the nondeterminism of concurrent execution. (3) Inter-procedural properties and function pointers need to be considered when finding SAC bugs. In this example, the function $x f r m \_p o l i c y \_a l l o c$ is called through $x f r m \_c o m p i l e \_p o l i c y$ referenced by a function pointer, after read_lock is called. In fact, a main reason why this bug persisted for a long time may be that it involves a function-pointer call.

Some recent studies $[19,60]$ have shown that SAC bugs have caused serious system hangs, and these bugs are often hard to locate and reproduce. Thus, to improve the reliability of the Linux kernel, it is necessary to design an effective approach to detect SAC bugs.

\section{CHALLENGES}

There are three main challenges in detecting SAC bugs in the Linux kernel.

\footnotetext{
${ }^{2}$ Patch link: http://lists.openwall.net/netdev/2010/08/13/47
} 


\subsection{C1: Accuracy and Efficiency in Code Analysis}

When identifying the code that may be executed in atomic context, the code analysis should be accurate and efficient. Flow-sensitive static analysis searches each code path with the goal of providing accuracy, but it is often inefficient in analyzing large software. The Linux kernel code base is very large (amounting to over 16M lines of source code, measured with CLOC [17], in our tested version Linux 4.17) and complex, and it contains many source files in multiple directories. Thus, directly using flow-sensitive analysis to check the whole Linux kernel may be quite time consuming.

\subsection{C2: Handling Function Pointers}

The Linux kernel is essentially an object-oriented system, in which data structures containing function pointers play the role of objects containing methods. This design improves extensibility, but greatly complicates static analysis. Indeed, many possible execution paths contain calls to function pointers, and static analysis needs to correctly determine the set of functions that may be referenced by these pointers. An accurate function pointer analysis is thus very important when detecting SAC bugs. On the one hand, identifying too few or no referenced functions implies that SAC bugs like the one shown in Figure 2 that hide in the missed functions cannot be detected. On the other hand, if incorrect functions are identified for function pointers, false SAC bugs may be reported.

\subsection{C3: Filtering out Repeated and False Bugs}

Flow-sensitive analysis may detect repeated bugs when these bugs take the spinlock at the same place and call the same sleep-able function but only differ in their code paths. Moreover, flowsensitive analysis may detect false bugs, because the analysis does not consider variable values when analyzing path conditions, and thus may search some infeasible code paths.

\section{KEY TECHNIQUES}

To solve the above challenges, we propose three key techniques. For $C 1$, we propose a summarybased flow-sensitive analysis to accurately and efficiently identify the code that may be executed in atomic context. For $C 2$, we propose a connection-based alias analysis to correctly identify the set of functions referenced by a function pointer. For $C 3$, we propose a path-check method to filter out repeated reports and false bugs. We now introduce these techniques.

\subsection{Summary-Based Analysis}

Our summary-based analysis identifies the code in atomic context, and it has the following properties: (1) The analysis is context-sensitive and inter-procedural, in order to maintain the spinlock status and detect atomic context across functions calls. (2) The analysis is flow-sensitive, in order to improve the accuracy. (3) The analysis uses function summaries to reduce repeated analysis, which can improve the efficiency. (4) The analysis uses a connection-based alias analysis (which will be introduced in Section 4.2) to handle function-pointer calls. (5) When the analysis encounters a call to a function that can sleep at runtime (a sleep-able function) in atomic context, the analysis uses a path-check method (which will be introduced in Section 4.3) to filter out repeated reports and false bugs.

Our summary-based analysis has two steps. The first step identifies interrupt handler functions and calls to spin-lock functions, which initiate atomic context. For interrupt handler functions, we identify the calls to interrupt-handler-register kernel interfaces (like request_irq and 

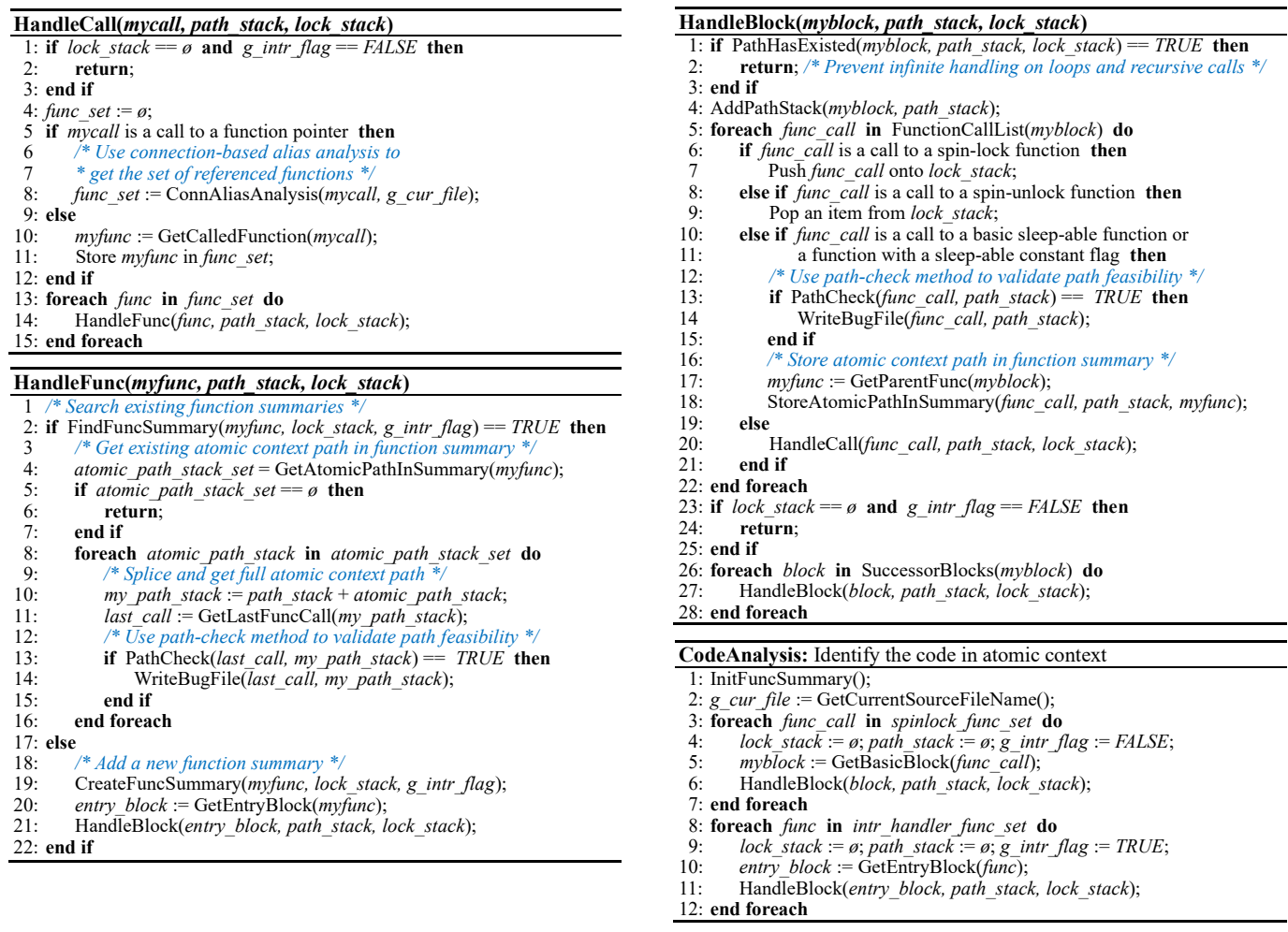

Fig. 3. Procedure of summary-based analysis.

tasklet_init in the Linux kernel), and extract interrupt handler functions from the relevant arguments. For calls to spin-lock functions, we identify them according to the function name.

The second step performs the main analysis, CodeAnalysis, which is defined in Figure 3. The analysis maintains two stacks, namely a path stack (path_stack) to store the executed code path (a sequence of locations of basic blocks and function calls) and a lock stack (lock_stack) to store the spinlock status. For simplicity, the analysis only considers the number and locations of locks in lock_stack, instead of the alias of each lock. The analysis also uses a global flag (g_intr_flag) to indicate whether the code is in an interrupt handler. If lock_stack is not empty or g_intr_flag is TRUE, the code may be executed in atomic context. Besides, the analysis creates and stores a function summary for each analyzed function for each execution context. This function summary contains the location of the function, the lock_stack and g_intr_flag for which the function is analyzed, and code paths of the SAC bugs found in the function (we refer to such a code path as an atomic-context code path). CodeAnalysis uses HandleCall to handle a function call, HandleBlock to handle a basic block, and HandleFunc to handle the definition of a called function.

HandleCall. It handles the function call mycall with the arguments path_stack and lock_stack. Firstly, HandleCall checks whether lock_stack is empty and g_intr_flag is FALSE (lines 1-3). If so, no spinlock is held and the code is not in an interrupt handler, and thus HandleCall returns. Secondly, HandleCall identifies the set of functions called by mycall (lines 5-12), which is represented by func_set. If mycall is a function-pointer call, HandleCall performs a connection-based alias analysis, described in Section 4.2, to identify the set of functions referenced by the function pointer, and 
stores them in func_set. Otherwise, HandleCall only stores the called function myfunc in func_set. Finally, HandleCall handles each function in func_set by using HandleFunc (lines 13-15).

HandleBlock. It handles the basic block myblock with the arguments path_stack and lock_stack. Firstly, HandleBlock searches path_stack to check whether myblock has been analyzed (lines 13) with respect to the current lock_stack. If so, it returns to avoid repeated analysis. Secondly, HandleBlock adds myblock into path_stack (line 4). Thirdly, HandleBlock handles each function call func_call in myblock (lines 5-22) in order. If func_call is a call to a spin-lock or spin-unlock function, HandleBlock respectively pushes the call onto or pops an item from lock_stack. If func_call is a call to a basic sleep-able function or a function with a sleep-able constant flag, HandleBlock uses a path-check method, described in Section 4.3, to validate whether the code path is feasible. If so, a SAC bug is found, and its call path is written into the bug report file. Then, HandleBlock stores the code path path_stack reaching func_call as an atomic-context code path in the summary of myblock's function myfunc, and this atomic-context code path is used to build the completed code path in the summary-based analysis. If func_call is not a call to a spin-lock, spin-unlock, basic sleep-able function or a function with a sleep-able constant flag, HandleBlock uses HandleCall to handle func_call. Fourthly, HandleBlock checks whether lock_stack is empty and g_intr_flag is FALSE (lines 23-25). If so, it returns. Finally, HandleBlock handles each successor basic block of myblock by using HandleBlock.

HandleFunc. It handles the function myfunc with the arguments path_stack and lock_stack. HandleFunc searches existing function summaries stored in the database, to check whether myfunc has been already handled under the current execution context (line 1). If so, HandleFunc uses the results produced by the previous analysis (lines 3-16). HandleFunc searches the function summary of myfunc to find atomic-context code paths starting from myfunc, and stores the code paths in a set atomic_path_stack_set. If no code path is found, namely myfunc contains no calls to sleep-able functions, then HandleFunc returns directly. Then, HandleFunc handles each code path atomic_path_stack in the set atomic_path_stack_set. It splices path_stack and atomic_path_stack to build a complete atomic context code path my_path_stack. Later, HandleFunc gets the last function call from my_path_stack as the end of the code path, and uses the path-check method, described in Section 4.3, to validate whether the code path is feasible. If so, a SAC bug is found, and its call path is written into the bug report file. If no function summary is found, it indicates that myfunc has not been handled under the current execution context. In this case, HandleFunc creates a new function summary for myfunc for the current execution context, stores the summary in the database, and analyzes the definition of myfunc starting from its entry basic block by using HandleBlock.

CodeAnalysis. It performs the main analysis, in three steps. Firstly, CodeAnalysis initializes the set of function summaries. Secondly, CodeAnalysis handles each call to a spin-lock function in the kernel code. It clears lock_stack and path_stack, and sets g_intr_flag to FALSE, and then starts the analysis by using HandleBlock to handle the basic block containing the call. Thirdly, CodeAnalysis handles each interrupt handler function. It clears lock_stack and path_stack, and sets g_intr_flag to TRUE, and then starts the analysis by using HandleBlock to handle the entry basic block of the function.

Our summary-based analysis has four main advantages: (1) By using flow-sensitive and contextsensitive analysis, our analysis can accurately identify the code that may be executed in atomic context. (2) By using function summaries and storing atomic-context code paths, our analysis can effectively reduce repeated analysis and utilize previous analysis results, to improve efficiency without damaging accuracy. (3) Our analysis is inter-procedural and can analyze functions across different source files and modules. (4) Our analysis handles function-pointer calls, which are not considered in existing approaches that detect SAC bugs $[15,24,46]$. However, a main limitation of 


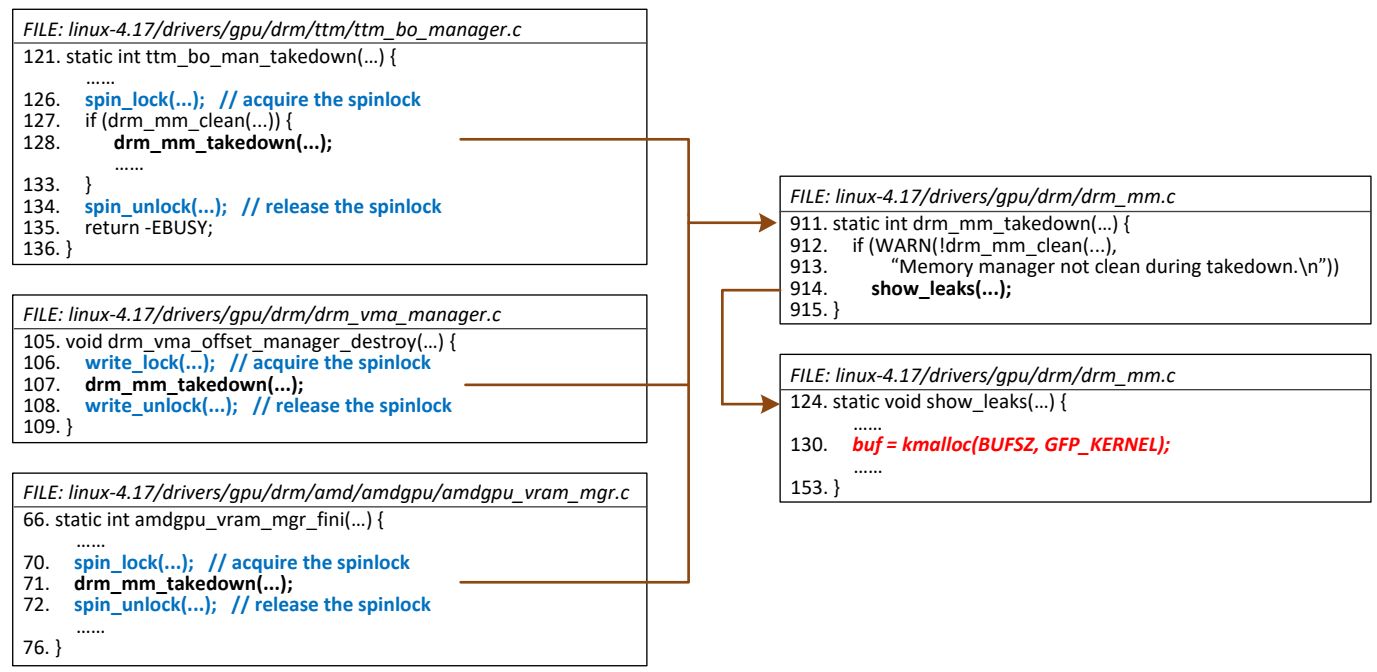

Fig. 4. Examples of summary-based analysis.

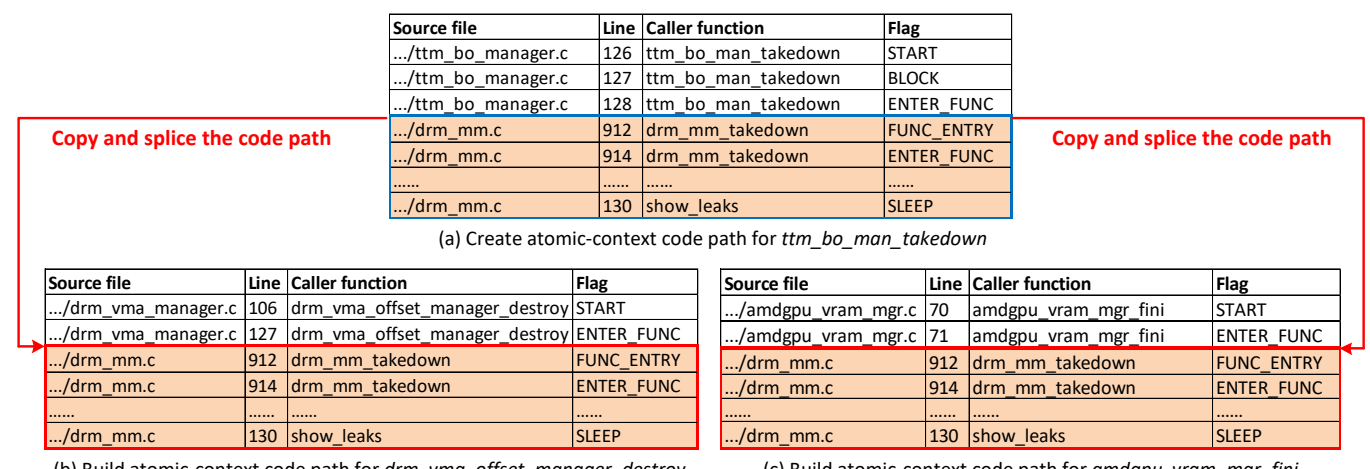

Fig. 5. Examples of atomic-context code paths.

our analysis is that variable value information is not considered, which may cause false positives in bug detection.

To illustrate our summary-based analysis, we consider the Linux kernel code shown in Figure 4. The functions ttm_bo_man_takedown, drm_vma_offset_manager_destroy and amdgpu_vram_mgr_fini all call the function drm_mm_takedown while holding a spinlock. The function drm_mm_takedown calls show_leaks, and the function show_leaks calls kmalloc with GFP_KERNEL, which can sleep at runtime resulting in three SAC bugs. In this example, the function drm_mm_takedown is called under the same lock status, namely holding one spinlock, thus this function can be just analyzed once, instead of three times.

For the example in Figure 4, assume that our summary-based analysis first handles the function ttm_bo_man_takedown, and creates the function summary for drm_mm_takedown while holding one spinlock. Our analysis stores the atomic-context code path in the function summary for drm_mm_takedown that is shown in Figure 5(a). Each item in the code path represents the code location of a basic block or function call that has to be traversed to reach the current point in the code. The item consists of four fields, namely the source file name, source line number, caller function 
and a flag. The flag indicates the analysis state of the code location. For example, in Figure 5, "START" means the location of the start of the analysis; "BLOCK" means the entry of a basic block; "ENTER_FUNC" means the location of a function call whose function definition is to be analyzed; "FUNC_ENTRY" means the entry of a function; "SLEEP" means the location of a sleepable function call. The called function and the flag are not strictly necessary for the algorithm, but facilitate generating an understandable report when a bug is found. When our analysis handles the functions drm_vma_offset_manager_destroy and amdgpu_vram_mgr_fini, it finds that the function drm_mm_takedown with holding one spinlock has been already analyzed, by searching the function summaries. Then, our analysis finds the atomic-context code paths stored in the summary of drm_mm_takedown. Later, as shown in Figure 5(b) and 5(c), our analysis copies this code path and splices it with the handled code paths in the functions drm_vma_offset_manager_destroy and amdgpu_vram_mgr_fini, to build complete atomic-context code paths. Finally, our analysis uses a path check method to perform code path validation, and finds that the code path is feasible. Thus, our analysis writes the bug information and code path into the bug report file.

\subsection{Connection-Based Function-Pointer Analysis}

Overall, we identify the set of functions called by a function-pointer call through two steps. Firstly, before the summary-based analysis, we collect the functions that are assigned to function pointers. Secondly, during the summary-based analysis, we select the correct function(s) at each given call site from the collected functions. The first step is performed straightforwardly, by scanning the assignments to function pointers in the Linux code. The second step, of how to identify the correct function(s) at a particular call site, is more difficult.

To illustrate the problem with the second step, we consider the Linux kernel code shown in Figure 6. Figure 6(a) shows that in the $e 1000$ driver, the function e1000_dump_eeprom calls a function pointer through the get_eeprom field of a struct ethtool_ops data structure (line 748). This field is initialized in multiple drivers to different functions. Figure 6(b) shows three candidate functions: e1000_get_eeprom, jme_get_eeprom and sky2_get_eeprom. Because this function pointer is called in the $e 1000$ driver, the referenced function(s) should be related to this driver. From the Makefile of the e1000 drivers shown in Figure 6(c), e1000_main.c is linked with e1000_ethtool.c in the same kernel module, thus e1000_get_eeprom should be the correct function referenced by the function pointer.

From this example, we observe that link information can be used to identify the correct function(s) referenced by a function-pointer call. The simplest case is that the candidate function and the function-pointer call are in the same source file. In other cases, for a candidate function $F$ that may be referenced by a function pointer, if its source file is linked with the source file of a function-pointer call, $F$ is very likely to be a correct referenced function for the function-pointer call. The link information reflects the connection between the source files of the candidate function and function-pointer call. This connection is strong, as the involved two source files are linked in the same kernel module.

Besides calling function pointers in the same kernel module, in the Linux kernel, one kernel module can also call the functions in another kernel module through function pointers. In this case, the link information is not sufficient to identify the correct function(s) referenced by a functionpointer call, because the related kernel modules may not be explicitly linked together. To handle this case, we observe that direct function calls can be used to identify the connection between the source files of the candidate function and function-pointer call. Specifically, if there exists a direct function call between File 1 and File2 $($ File $1 \rightarrow$ File 2 or File $2 \rightarrow$ File1), then we say that File 1 and File 2 have a direct connection. This connection can be transitive. Namely, if there exists a direct function call from File1 to File2 (File1 $\rightarrow$ File2) and from File2 to File3 (File2 $\rightarrow$ File3), then we say that File1 and File 3 have an indirect connection (File1 $\rightarrow$ File3). This connection of function call is weaker than 


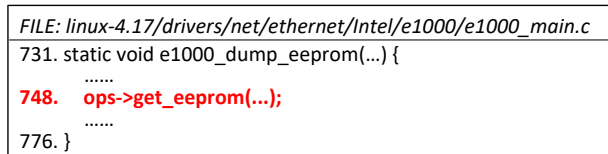

(a) Function pointer call.

FILE: linux-4.17/drivers/net/ethernet/Intel/e1000/Makefile obj-\$(CONFIG_E1000) $+=$ e1000.0

e1000-objs := e1000 main.o e1000 hw.o e1000_ethtool.o e1000 param.o

(c) Makefile for the 1000 driver.

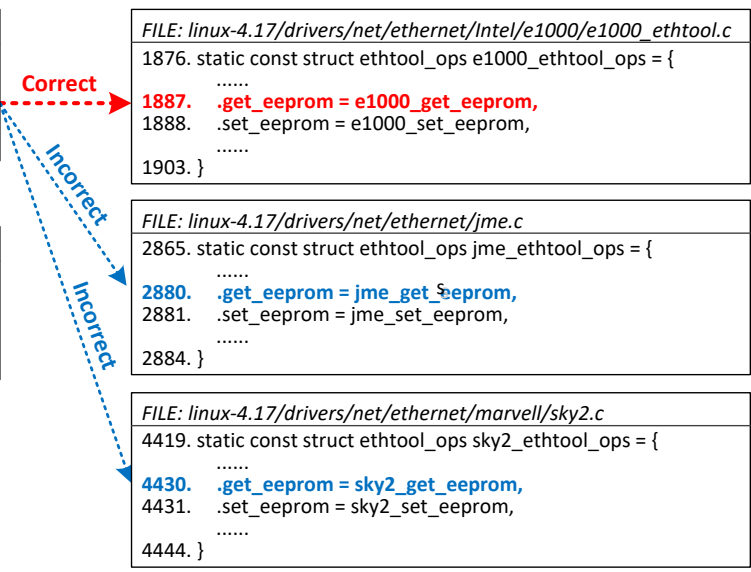

(b) Some functions that may be referenced by the function pointer

Fig. 6. Examples of handling function-pointer calls through link information.

\begin{tabular}{|c|c|c|}
\hline FILE: linux-4.17/drivers/scsi/libfc/fc_lport.c & \multirow[b]{3}{*}{ Function pointer } & FILE: linux-4.17/drivers/scsi/fcoe/fcoe_ctlr.c \\
\hline 729. static void fc_Iport_enter_ready(...) \{ & & 3189. static void fcoe_ctrl_mode_set(...) \{ \\
\hline $\begin{array}{l}\text { 739. if (!lport->ptp_rdata) } \\
\text { 740. Iport->tt.disc_start(...); } \\
\text { 741. }\end{array}$ & & $\begin{array}{l}\text { 3200. Iport->tt.disc_recv_req }=\text { fcoe_ctlr_disc_recv; } \\
\text { 3201. Iport->tt.disc_start }=\text { fcoe_ctlr_disc_start; } \\
\text {...... }\end{array}$ \\
\hline 1868. int fc Iport init(...) \{ & & 3216. \\
\hline 1893. $\}^{\cdots \cdot . \cdot}$ & & $\begin{array}{l}\text { 3227. int fcoe_libfc_config }(. . .)\{ \\
\ldots \ldots .\end{array}$ \\
\hline & & 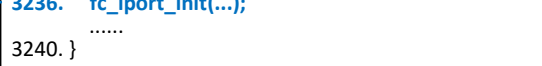 \\
\hline
\end{tabular}

(a) Example of function pointer call through a direct function-call connection.

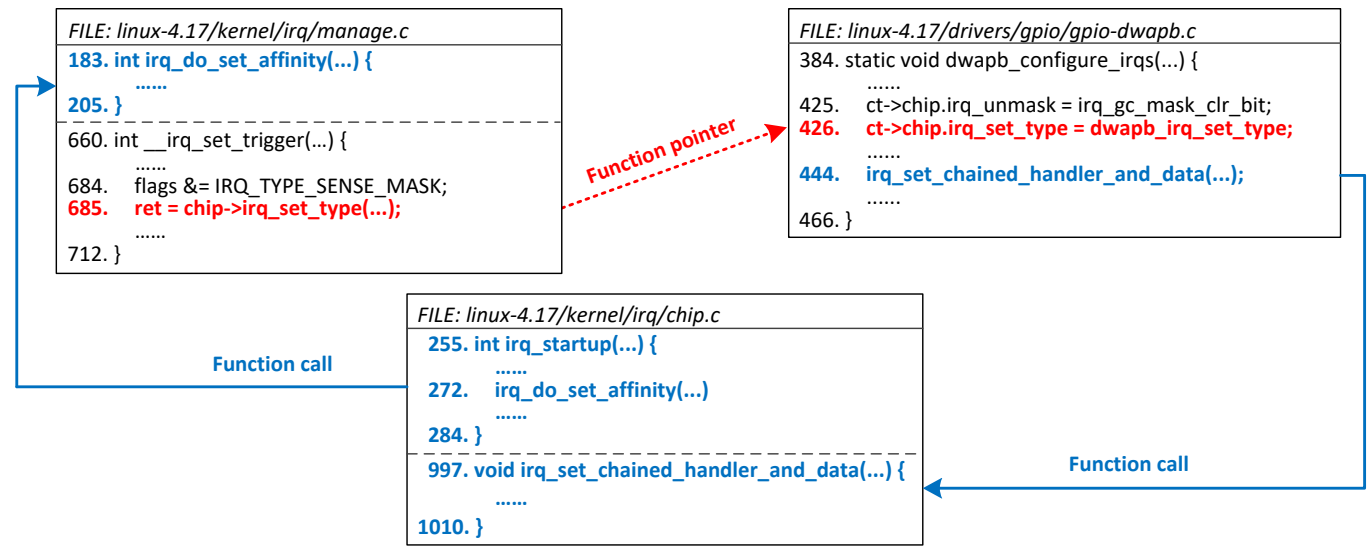

(b) Example of function pointer call through an indirect function-call connection.

Fig. 7. Examples of handling function-pointer calls through function-call connections.

the link-information connection, as the involved two source files are in different kernel modules. For this reason, the link-information connection is preferentially used for function-pointer analysis.

To illustrate the function-call connection between different kernel modules, we consider two examples in the Linux kernel code shown in Figure 7. 
Figure 7(a) shows an example of a direct function-call connection. The driver libfc uses a functionpointer call on line 740 in $f c_{-}$lport.c, and the driver $f$ coe has an assignment of this function pointer to fcoe_ctlr_disc_start on line 3201 in fcoe_ctlr.c. In fcoe_ctlr.c, the function fcoe_libfc_config calls the function $f_{c}$ _lport_init defined in $f c_{-} l$ port.c, thus the two source files have a direct connection $\left(f\right.$ coe_ctlr.c $\left.\rightarrow f c \_l p o r t . c\right)$. For this reason, fcoe_ctlr_disc_start in fcoe_ctlr.c should be a function referenced by the function-pointer call in $f c_{-}$lport.c.

Figure 7(b) shows an example of an indirect function-call connection. The kernel uses a functionpointer call on line 685 in manage.c, and the driver gpio has an assignment of this function pointer to dwapb_irq_set_type on line 426 in gpio-dwapb.c. In gpio-dwapb.c, there is no function that calls a function defined in manage.c, so the two source files do not have a direct function-call connection. However, the function dwapb_configure_irqs calls the function irq_set_chained_handler_and_data defined in chip.c (gpio-dwapb.c $\rightarrow$ chip.c), and in chip.c, the function irq_startup calls the function irq_do_set_affinity defined in manage.c (chip.c $\rightarrow$ manage.c), so gpio-dwapb.c and manage.c have an indirect function-call connection (gpio-dwapb.c $\rightarrow$ chip.c). For this reason, dwapb_irq_set_type in gpio-dwapb.c should be a function referenced by the function-pointer call in manage.c.

Based on the connections of link information and function calls, we propose a connection-based alias analysis to identify the set of functions referenced by a function pointer. Specifically, our analysis has two steps:

The first step is to collect candidate functions referenced by function pointers and the connections between source files. This step is performed before the summary-based analysis described in Section 4.1. To collect candidate functions, our analysis traverses the Linux kernel code and handles functionpointer assignments. Specifically, our analysis focuses on function-pointer assignments involving data-structure fields, for two reasons. Firstly, many function pointers in kernel code are obtained from data-structure fields [9], especially in device drivers, as illustrated in Figure 6 and Figure 7. We have studied the function-pointer calls in the x 86 code of Linux 4.17, and find that over 95\% (i.e., 34,452 out of 36,174 ) involve functions stored in data-structure fields. Secondly, a function pointer stored in a data-structure field can be explicitly can be distinguished from other function pointers with the data-structure type, field offset and function-pointer type. To collect the connections between source files, our analysis records the link information during kernel code compilation and records direct function calls between different source files. All of this information is stored in the database.

The second step is to identify the set of functions referenced by a function pointer, which is done during the summary-based analysis described in Section 4.1. Figure 8 shows the main procedure FuncPtrAnalysis. The inputs are a function-pointer call func_ptr_call and the name of its source file $s r c$ file where the summary-based analysis starts. The output is func_set, the set of functions referenced by the function pointer for func_ptr_call. Firstly, our analysis clears func_set, and gets the called function pointer func_ptr of func_ptr_call (lines 1-2). Secondly, by looking up the collected information stored in the database, our analysis gets the set of candidate functions for func_ptr, which is represented as cand_func_set (line 3). Because our analysis focuses on function pointers stored in data-structure fields, we perform field-based analysis [30] here. Specifically, If func_ptr is a function pointer stored in a data-structure field, we get its candidate functions according to its data-structure type, field offset and function-pointer type. Thirdly, our analysis selects the functions from cand_func_set whose source file cand_src_file has a link-information connection with src_file (lines 4-9). The selected functions are put in func_set. Because a link-information connection is strong, if any function is selected through this connection, our analysis returns func_set and ends (lines 10-12). Otherwise, our analysis selects the functions from cand_func_set whose source file 


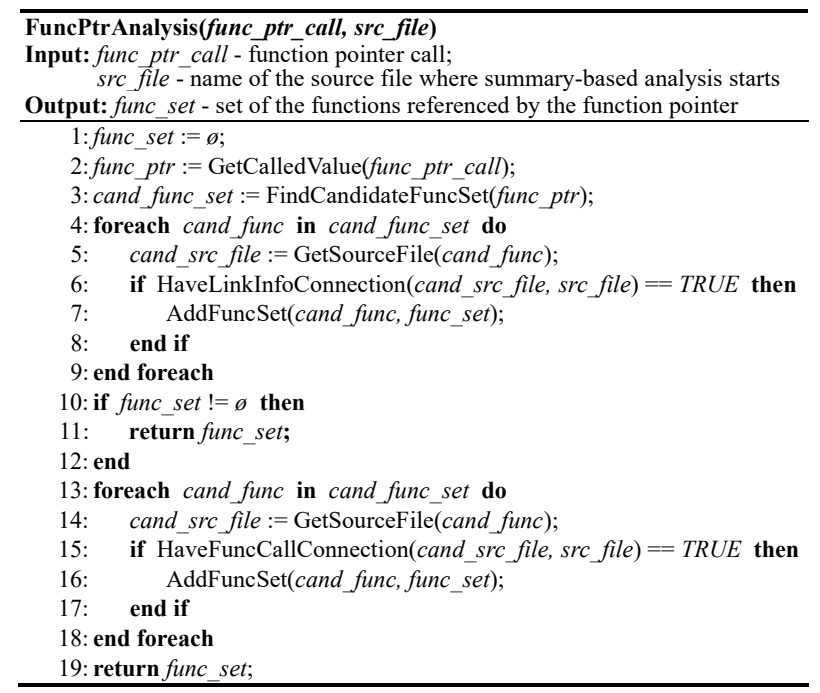

Fig. 8. Procedure of connection-based function-pointer analysis.

cand_src_file has a function-call connection with src_file (lines 13-18). The selected functions are put in func_set. Finally, our analysis returns func_set and ends (line 19).

The main advantage of our connection-based alias analysis is to improve the accuracy of identifying correct referenced functions for function-pointer calls. This advantage can benefit the detection of SAC bugs in two aspects: (1) Deep SAC bugs involving function pointers can be detected. (2) Filtering out incorrect functions for function pointers reduces the false positive rate. Another advantage of our alias analysis is high efficiency, because the analysis is flow-insensitive and its processing is not complex. This advantage is quite beneficial in analyzing code of millions of lines, as found in the Linux kernel. However, due to flow insensitivity, the identified function(s) may be incorrect in a specific control flow. Moreover, in the current implementation, our analysis focuses on function pointers stored in data-structure fields, and other kinds of function pointers are not handled, so some SAC bugs involving these unhandled function pointers may be missed.

At present, our connection-based alias analysis is only used to detect SAC bugs. In another work [5], we have developed the tool DCNS that detects the inverse of SAC bugs, where non-sleep operations are used unnecessarily, outside of atomic context. That work uses a simpler version of connection-based alias analysis that only considers function call connections and does not take into account link information. In both of these cases, our alias analysis only handles function-pointer calls that are considered to occur in atomic context. We are currently extending our alias analysis to detect other kinds of bugs involving function-pointer calls.

\subsection{Path-Check Bug Filtering}

Given the code paths recorded during our summary-based analysis, we use a path-check method to filter out repeated reports and false bugs.

Firstly, our method filters out repeated bugs. For each new possible bug, our method checks whether its starting and ending basic blocks are the same as those of an already detected bug, and whether the ending basic block uses the same sleep-able function call. If the two conditions are both satisfied, the possible bug is marked as a repeated bug and is filtered out. 


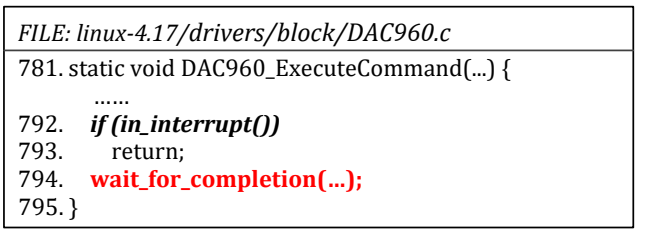

(a) Specific kernel interface.

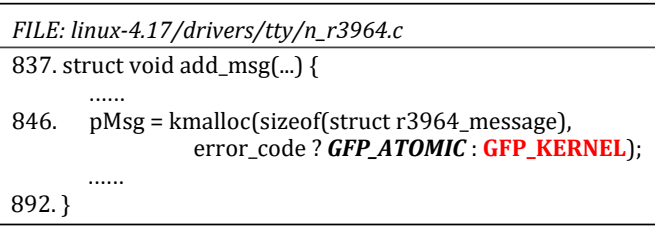

(b) Non-sleep constant flag.

Fig. 9. Examples of code styles for avoiding SAC bugs.

Secondly, our method filters out false bugs, which are mainly introduced by the fact that our summary-based analysis neglects variable value information. Our basic strategy is to validate the path conditions [12] of the code path for each possible bug. As shown in Figure 4, the code path consists of nodes, and each node can be a basic block or a function call whose called function is analyzed. Our method scans each node in the code path in order, records the stored value of each variable in each basic block, and validates the branch condition of each basic block according to the recorded values of related variables. If the validation fails, the possible bug is marked as a false bug and is filtered out.

Besides, by studying the Linux kernel code, we also find two common coding styles used by kernel developers to avoid introducing SAC bugs: (1) A function call to a specific kernel interface (like in_interrupt) is used to check for atomic context. (2) A non-sleep constant flag (like GFP_ATOMIC) is used as an argument under a condition in the function call. Figure 9 shows two examples in the Linux kernel code. In Figure 9(a), a conditional tests the result of calling in_interrupt to check whether the code is executed in an interrupt handler and decide whether the sleep-able function wait_for_completion can be called. In Figure 9(b), the call to the function kmalloc chooses between using a non-sleep constant flag GFP_ATOMIC or a sleep-able constant flag GFP_KERNEL as an argument. In fact, these two coding styles indicate semantic information, namely that the code can be in atomic context or non-atomic context. If a code path associated with a possible bug satisfies either of these two code styles, the possible bug is marked as a false bug and is filtered out.

\section{APPROACH}

Based on the three techniques in Section 4, we propose a static approach DSAC, to effectively detect SAC bugs in the Linux kernel. We have implemented DSAC using the Clang compiler [16], and perform static analysis on the LLVM bytecode of each source file. Figure 10 shows the architecture of DSAC, which has five parts:

- Code compiler. This part compiles each source file of the Linux kernel into an LLVM bytecode file, and records link information during code compilation and linking.

- Information collector. This part analyzes each LLVM bytecode file to collect useful information about the code, and stores the information in a MySQL database [43]. This information will be used in the remaining analyses.

- Connection extractor. This part uses the collected code information and link information to extract connections between source files, and stores them in the MySQL database.

- Bug detector. This part performs our summary-based analysis to detect possible SAC bugs from the LLVM bytecode.

- Bug filter. This part uses our path-check method to filter out repeated and false bugs, and generates reports for the final detected SAC bugs.

Based on this architecture, DSAC consists of four phases, which will be introduced in Sections 5.1-5.4. Section 5.5 will analyze the parallelism of these phases. 


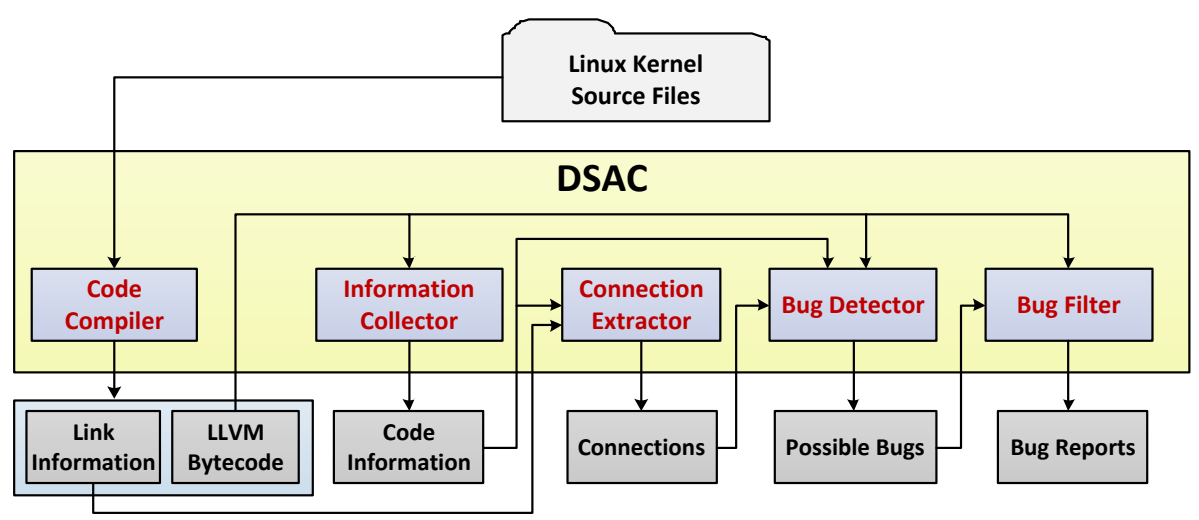

Fig. 10. DSAC architecture.

\subsection{Code Compilation}

In this phase, the code compiler performs code compilation and linking using the Clang compiler, in three steps: (1) compile each source file into a LLVM bytecode file; (2) compile each bytecode file into an assembly file and then into an object file; (3) link one or more object files together into a kernel object file, representing a loadable kernel module.

The code compiler keeps the set of LLVM bytecode files generated in the first step for the remaining analyses, and records the link information obtained from the third step.

\subsection{Information Collection}

In this phase, by analyzing LLVM bytecode files, the information collector extracts and stores useful information about the code of the Linux kernel, including the name and position of each function definition, the functions that may be referenced by each function pointer, and the callee and caller functions of each direct function call.

\subsection{Connection Extraction}

In this phase, the connection extractor extracts connections between source files. To extract linkinformation connections, the extractor analyzes the recorded link information, to get the source files that are linked together. To extract function-call connections, the extractor analyzes each function call between two different source files, and records the information that these two source files have a direct function-call connection.

\subsection{Bug Detection}

In this phase, the bug detector first performs summary-based analysis on the LLVM bytecode files, using the collected code information. To handle function-pointer calls, the detector performs connection-based alias analysis to identify the set of functions referenced by the function pointer, and processes each function in the resulting set. Then, the bug filter filters out repeated reports and false bugs. Finally, DSAC produces detailed reports for the found SAC bugs (including code paths and source file names), to help the user to locate the bugs.

\subsection{Parallelism}

The first three phases each work on the individual files independently, benefiting from the information collected in the previous phases. Thus, these phases can be parallelized straightforwardly. 
Table 1. Evaluated Linux kernel versions

\begin{tabular}{|l|c|c|}
\hline Description & Linux 3.17.2 & Linux 4.17 \\
\hline Release time & October 2014 & June 2018 \\
\hline Source files(.c) & $22.8 \mathrm{~K}$ & $25.9 \mathrm{~K}$ \\
\hline Source code lines & $12.4 \mathrm{M}$ & $16.9 \mathrm{M}$ \\
\hline
\end{tabular}

In the fourth phase, the summary-based analysis traces through function calls, which may cross file boundaries, when the called function is defined in another file and no function summary is available for the current analysis. In this case, we can also run the analysis in parallel, relying on the global database of function summaries to prevent repeated analysis of functions in the same context across different threads.

\section{EVALUATION}

\subsection{Experimental Setup}

To validate the effectiveness of DSAC, we evaluate it on the Linux kernel code. To cover different kernel versions, we select an old version 3.17.2 (released in October 2014) and a recent version 4.17 (released in June 2018). Table 1 shows some information about these kernel versions (the lines of source code are measured with CLOC [17]). We run the experiments on a Lenovo x86-64 PC with four Intel i5-3470@3.20G processors and 8GB memory. We compile the kernel code using Clang 6.0. We use the kernel configuration allyesconfig to enable all code for the $\mathrm{x} 86$ architecture. Because DSAC can work in parallel, we configure DSAC to run on four threads.

To run DSAC, we perform three steps. Firstly, we configure DSAC for the Linux kernel, by providing the names of 29 pairs of spin-lock and spin-unlock functions (such as spin_lock_irq and spin_unlock_irq), 3 interrupt-handler-register functions (request_irq, tasklet_init and devm_request_irq), 24 basic sleep-able kernel interfaces (such as msleep and usleep_range) and 5 sleep-able flags (such as GFP_KERNEL and GFP_USER). Secondly, we execute DSAC's compiling script to compile the source code of the Linux kernel. Finally, we execute DSAC's bug-detection script to detect SAC bugs. The second and third steps are fully automated.

\subsection{Bug Detection}

To validate whether DSAC can find known bugs, we use DSAC to check the Linux 3.17.2 kernel source code. To validate whether DSAC can find new bugs, we use DSAC to check the Linux 4.17 kernel source code. To check the accuracy of the bug-detection results, we manually check all detected bugs to identify whether they are real bugs. Table 2 shows the results. In this table, column "DSAC" shows the results of DSAC, and column "DSAC_noptr" shows the results of a variant of DSAC that does not consider the sets of functions possibly invoked by function-pointer calls. From Table 2, we have the following findings:

(1) DSAC can scale to large code bases. In Linux 3.17.2 and 4.17, DSAC respectively handles the $11.7 \mathrm{~K}$ and $16.7 \mathrm{~K}$ source files included in the $\mathrm{x} 86$ allyesconfig Linux kernel configuration. These files contain 7.0M and 9.8M lines of source code, as measured with CLOC [17]. DSAC starts its analysis from many entry basic blocks and many interrupt handler (INTR) functions. Still, some source files of the Linux kernel are not handled by DSAC, because they are not enabled for the x86 architecture.

(2) DSAC effectively handles many function-pointer calls, namely it successfully identifies the referenced functions of many function-pointer calls. DSAC respectively handles $73 \%$ and $67 \%$ of all encountered function-pointer calls in Linux 3.17.2 and 4.17. Among these handled function-pointer calls, $85 \%$ and $83 \%$, respectively, are handled by link-information connections. 
Table 2. Bug detection results.

\begin{tabular}{|c|c|c|c|c|c|}
\hline \multirow{2}{*}{\multicolumn{2}{|c|}{ Description }} & \multicolumn{2}{|c|}{ Linux 3.17.2 } & \multicolumn{2}{|c|}{ Linux 4.17} \\
\hline & & DSAC & DSAC_noptr & DSAC & DSAC_noptr \\
\hline \multirow{2}{*}{ Code handling } & Source files (.c) & $11.7 \mathrm{~K}$ & $11.7 \mathrm{~K}$ & $16.7 \mathrm{~K}$ & $16.7 \mathrm{~K}$ \\
\hline & Source code lines & $7.0 \mathrm{M}$ & $7.0 \mathrm{M}$ & $9.8 \mathrm{M}$ & $9.8 \mathrm{M}$ \\
\hline \multirow{4}{*}{$\begin{array}{l}\text { Summary-based } \\
\text { analysis }\end{array}$} & Handled INTR functions & 966 & 966 & 1424 & 1424 \\
\hline & Entry basic blocks & 42190 & 42190 & 50929 & 50929 \\
\hline & Handled functions & $51 \mathrm{~K}$ & $37 \mathrm{~K}$ & $65 \mathrm{~K}$ & $47 \mathrm{~K}$ \\
\hline & Function summaries & $79 \mathrm{~K}$ & $52 \mathrm{~K}$ & $103 \mathrm{~K}$ & $69 \mathrm{~K}$ \\
\hline \multirow{6}{*}{$\begin{array}{l}\text { Function-pointer } \\
\text { analysis }\end{array}$} & Encountered function-pointer calls & 14185 & - & 17349 & - \\
\hline & Handled function-pointer calls & 10323 & - & 11915 & - \\
\hline & Calls handled by link-information connection & 8741 & - & 9859 & - \\
\hline & Calls handled by function-call connection & 1582 & - & 2056 & - \\
\hline & Candidate referenced functions & $113 \mathrm{~K}$ & - & $138 \mathrm{~K}$ & - \\
\hline & Identified referenced functions & $40 \mathrm{~K}$ & - & $45 \mathrm{~K}$ & - \\
\hline \multirow{4}{*}{ Bug detection } & Filtered repeated bugs & 22687 & 5151 & 28374 & 11586 \\
\hline & Filtered false bugs & 7796 & 4284 & 9957 & 5141 \\
\hline & Final detected bugs & 891 & 464 & 1159 & 615 \\
\hline & Real bugs & 805 & 432 & 1068 & 564 \\
\hline \multirow{4}{*}{ Time usage } & P1: Information collection & $17 \mathrm{~m}$ & $17 \mathrm{~m}$ & $24 \mathrm{~m}$ & $24 \mathrm{~m}$ \\
\hline & P2: Connection extraction & $8 \mathrm{~m}$ & $0 \mathrm{~m}$ & $11 \mathrm{~m}$ & $0 \mathrm{~m}$ \\
\hline & P3: Bug detection & $53 \mathrm{~m}$ & $23 \mathrm{~m}$ & $62 \mathrm{~m}$ & $28 \mathrm{~m}$ \\
\hline & Total & $78 \mathrm{~m}$ & $40 \mathrm{~m}$ & $97 \mathrm{~m}$ & $52 \mathrm{~m}$ \\
\hline
\end{tabular}

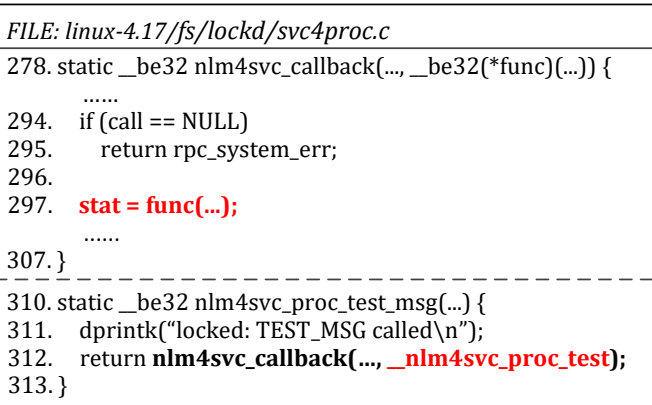

(a) Function pointer of function argument.

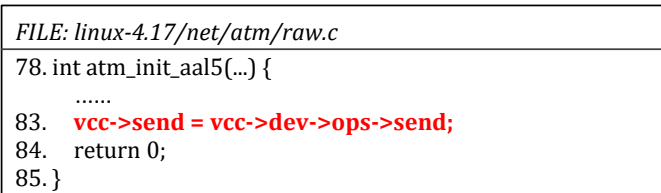

FILE: linux-4.17/net/atm/clip.c

327. static netdev_tx_t clip_start_xmit(...) \{

395. $\quad$ vcc->send(...);

.......

413. $\}$

(b) Function pointer of indirect assignment.

Fig. 11. Examples of unhandled function-pointer calls by DSAC.

The remaining $27 \%$ and $33 \%$ of function-pointer calls are not handled. There are two cases that are commonly not covered. Firstly, DSAC focuses on function pointers stored in data-structure fields, and cannot handle the function pointers that are not referenced in this way. For example in Figure 11(a), on line 312, the function nlm4svc_proc_test_msg calls the function nlm4svc_callback with an argument that is a pointer to the function __nlm4svc_proc_test. And the function nlm4svc_callback calls__nlm4svc_proc_test via the pointer argument on line 297. Because the function pointer on line 297 is not a data-structure field, DSAC cannot identify the referenced functions for this function pointer call. Secondly, some function pointers stored in data-structure fields are not directly assigned to explicit functions, and DSAC cannot identify the functions involved in such function-pointer assignments. For example in Figure 11(b), the function pointer vcc->send is assigned through another function pointer vcc->dev->ops->send on line 83, so the candidate referenced functions of $\mathrm{Vcc}->$ send cannot be directly identified only according to this assignment. For this reason, DSAC cannot identify the referenced functions for the function-pointer call to vcc->send on line 395. 
(3) Our connection-based alias analysis is effective in improving the accuracy of function-pointer analysis. It retains around $35 \%$ and $33 \%$ of the candidate referenced functions in Linux 3.17 .2 and 4.17, respectively. The remaining $65 \%$ and $67 \%$ candidate referenced functions are discarded because they are considered to be incorrect in the calling context of the given function-pointer call. By discarding these incorrect functions, DSAC reduces unnecessary analysis of atomic context, and also reduces the number of false positives in bug detection.

(4) Our path-check method is effective in filtering out repeated reports and false bugs. It filters out more than $95 \%$ of all possible bugs, which are considered to be repeated reports and false positives according to their code paths. Among the false bugs filtered out by DSAC, $28 \%$ and $25 \%$ are related to coding styles about atomic context (such as using the function call to in_interrupt and the non-sleep constant flag GFP_ATOMIC).

(5) DSAC is effective in bug detection with or without function-pointer analysis:

For Linux 3.17.2, DSAC reports 891 bugs, of which we have identified 805 as real bugs. Two experienced researchers each spent six days manually checking these bugs. Among these bugs, 304 bugs have been fixed in Linux 4.17. Without function-pointer analysis (DSAC_noptr), it reports 464 bugs, of which we have identified 432 as real bugs. Among these bugs, 171 bugs have been fixed in Linux 4.17. The results indicate that DSAC can indeed find known bugs.

For Linux 4.17, DSAC reports 1159 bugs, of which we have identified 1068 as real bugs. Two experienced researchers each spent eight days manually checking these bugs. Without functionpointer analysis (DSAC_noptr), it reports 615 bugs, of which we have identified 564 bugs as real bugs. Among the 1068 real bugs found by DSAC, we randomly selected 300 and reported them to kernel developers. As of September 2018, 220 bugs have been confirmed. We also sent 94 patches to fix 208 of the reported bugs, and 51 patches fixing 115 bugs have been applied by the kernel maintainers. The results indicate that DSAC can indeed find new bugs.

(6) Using function-pointer analysis, DSAC can find many deep bugs that involve function-pointer calls. In Linux 3.17.2 and 4.17, DSAC respectively finds 341 and 505 more real bugs than that without function pointer analysis (DSAC_noptr), accounting for $42 \%$ and $47 \%$ of all real found bugs.

(7) DSAC achieves good accuracy in bug detection. The false positive rates are respectively only 9.7\% and 7.9\% in Linux 3.17.2 and 4.17. Without function-pointer analysis (DSAC_noptr), the false positive rates are respectively only $6.9 \%$ and $8.3 \%$.

(8) DSAC is efficient in code analysis. On four running threads, it respectively spends 78 and 97 minutes on handling $11.7 \mathrm{~K}$ and $16.7 \mathrm{~K}$ source files in Linux 3.17.2 and 4.17. Without function-pointer analysis, on four running threads, DSAC respectively spends 40 and 52 minutes of running time.

\subsection{Example Bugs}

Figure 12 shows some real bugs found by DSAC in Linux 4.17, that have been confirmed by kernel developers. In Figure 12(a), the socionext driver registers the function ave_irq_handler as an interrupt handler using the kernel interface request_irq on line 1236. The function ave_irq_handler calls ave_rxfifo_reset on line 932, which calls a sleep-able function usleep_range on lines 888 and 892 , causing two SAC bugs. ${ }^{3}$

In Figure 12(b), the function __setup_irq calls raw_spin_lock_irqsave to acquire a spinlock, and then calls __irq_set_trigger on line 1353. This called function uses a function pointer call chip->irq_set_type on line 685. In the adp5588 driver, this function pointer is assigned to the function adp5588_irq_set_type on line 237. This function calls adp5588_gpio_direction_input on line 224, which calls a sleep-able function mutex_lock on line 113, causing a SAC bug. ${ }^{4}$

\footnotetext{
${ }^{3}$ Link: https://lore.kernel.org/patchwork/patch/986431/

${ }^{4}$ Link: https://lkml.org/lkml/2018/8/13/197
} 


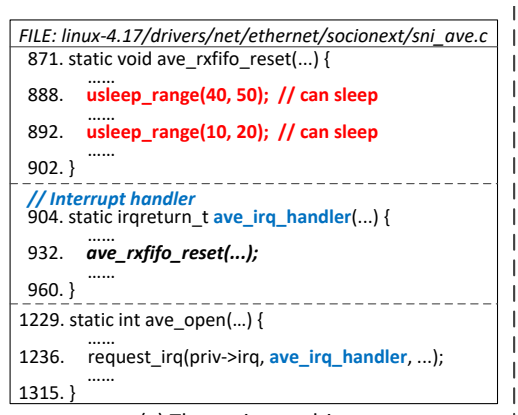

(a) The socionext driver.

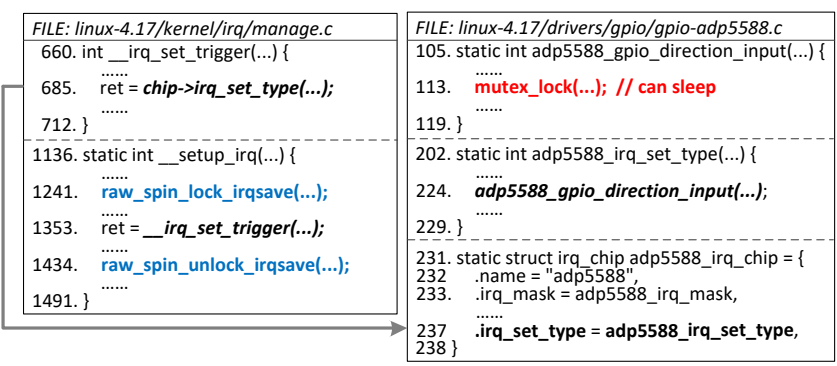

(b) The adp5588 driver.

Fig. 12. Examples of real bugs found by DSAC.

Figure 13 shows the bug reports for these bugs generated by DSAC. The report explicitly shows the function call path (from bottom to top) of the detected bug, including the source file's name, the source line number, the callee function's name and the caller function's name. In the report, "[FUNC_PTR]" means that a function-pointer call is used. According to the bug report, the user can conveniently locate the detected bug.

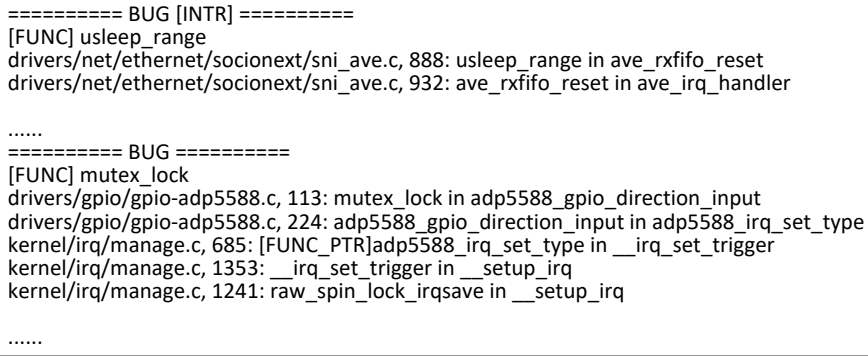

Fig. 13. Example of bug reports generated by DSAC.

\subsection{Bug Characteristics}

Reviewing the SAC bugs found by DSAC, we find some interesting characteristics of them:

(1) Most of the detected bugs (1056 in Linux 4.17) involve multiple function calls. Indeed, kernel developers may easily forget that the code is in atomic context across multiple function calls, especially function-pointer calls.

(2) Many of the detected bugs (313 in Linux 4.17) involve multiple parts of the kernel. For example in Figure 12(b), the SAC bug involves two parts of the kernel, namely the interrupt handling manager and the driver adp5588. Figure 14 shows another representative bug found by DSAC and the generated bug report. This SAC bug involves three drivers of different classes, namely input, $m f d$ and spi drivers. Indeed, when calling functions defined in other parts of the kernel, kernel developers may easily forget to check whether these functions can sleep and just call these functions according to their functionality.

(3) Many of the detected bugs (235 in Linux 4.17) are related to resource allocation. For example, many SAC bugs are caused by allocating a resource using a sleep-able flag GFP_KERNEL (like the bugs shown in Figure 4), which allows sleeping and waiting until the resource becomes available. 


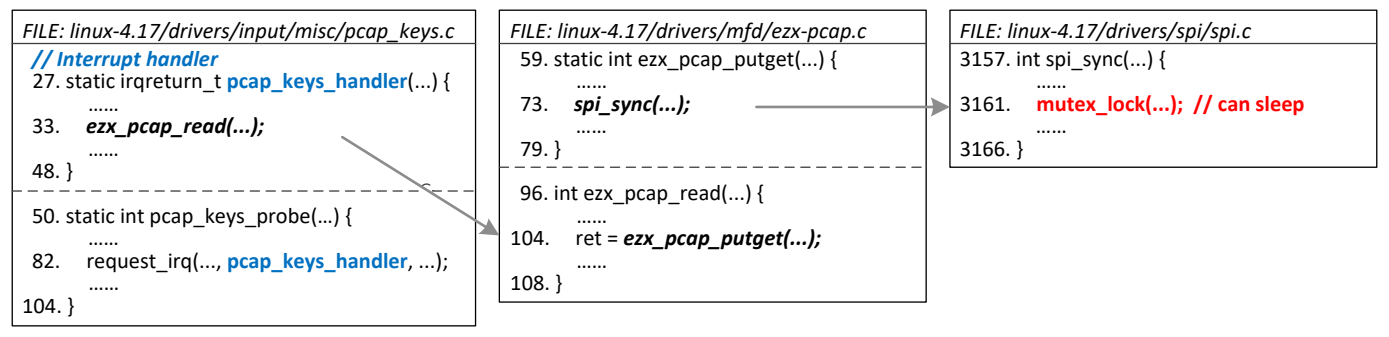

$$
\begin{aligned}
& =========\text { BUG [INTR] ========== } \\
& \text { [FUNC] mutex_lock } \\
& \text { drivers/spi/spi.c, 3161: mutex_lock in spi_sync } \\
& \text { drivers/mfd/ezx-pcap.c, 73: spi_sync in ezx_pcap_putget } \\
& \text { drivers/mfd/ezx-pcap.c, 104: ezx_pcap_putget in ezx_pcap_read } \\
& \text { drivers/input/misc/pcap_keys.c, 33: ezx_pcap_read in pcap_keys_handler }
\end{aligned}
$$

Fig. 14. Example SAC bug involving multiple parts of the kernel and its bug report.

(4) Multiple detected bugs are caused by calling the same sleep-able kernel interfaces. This characteristic indicates that some commonly used kernel interfaces may be supposed to be non-sleep by some kernel developers, but these kernel interfaces can sleep in reality. An example is that the three bugs shown in Figure 4 are all caused by calling the sleep-able kernel interface drm_mm_takedown. Besides, in DSAC's bug reports, hid_hw_request (causing 18 bugs) and clk_get_rate (causing 6 bugs) are also such sleep-able kernel interfaces.

(5) Few of the detected bugs (152 in Linux 4.17) are in interrupt handlers. Indeed, driver developers often write clear comments to mark the driver functions that are called from an interrupt handler, to protect against calling sleep-able functions in these functions.

\subsection{Bug Distribution}

We classify the detected bugs according to the directory of their source files. As described in Section 6.4, many detected bugs involve multiple parts of the kernel, so the bugs are classified by the source file of the starting basic block (containing the call to a spin-lock function or the entry of an interrupt handler function) in the bug report. Figure 15 shows the results for the real bugs identified by our manual check.

We find that drivers have more than $77 \%$ of all detected real bugs. This is somewhat higher than the percentage of code represented by drivers (67\% in Linux 4.17). Thus, drivers remain a significant source of system problems [52]. We also classify the detected bugs in drivers by the driver class. We find that network, scsi and staging drivers together have more than $50 \%$ of the bugs in drivers. A possible reason is that these drivers also have much more code than the other driver classes.

\subsection{False Positives}

False positives are mainly introduced in two cases:

Firstly, due to flow insensitivity, our connection-based alias analysis may identify incorrect referenced functions for function pointers. Figure 16(a) shows a false reported bug in such a case. The function qla2100_intr_handler acquires a spinlock on line 59, and then uses a function-pointer call ha->isp_ops->fw_dump on line 77 . This function pointer has several candidate referenced functions, such as qla2100_fw_dump and qla8044_fw_dump. Because the two functions are respectively defined in qla_dbg.c and qla_nx2.c, which are both linked with qla_isr.c and qla_os.c according to the Makefile, our alias analysis identifies both of the functions as referenced functions of the function-pointer call. But according to the data flow of the source file, qla2100_fw_dump should 

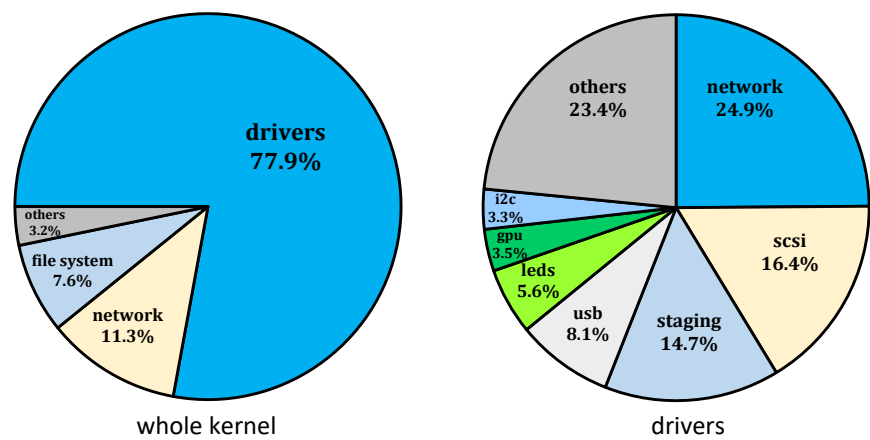

(a) Linux 3.17.2.
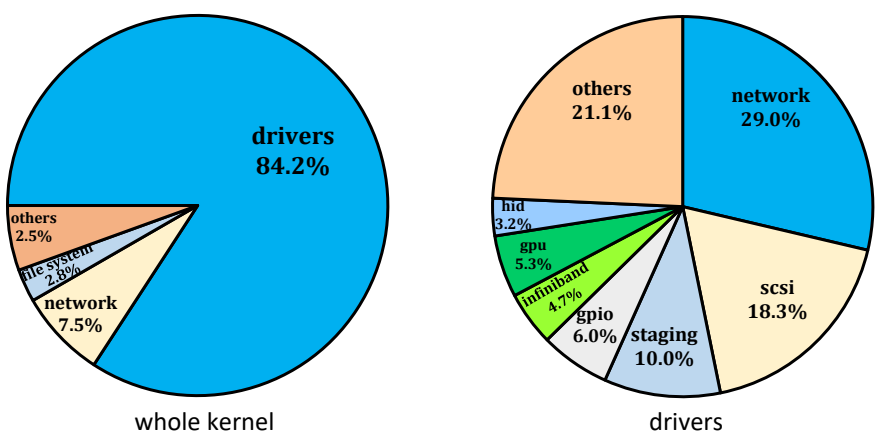

(b) Linux 4.17 .

Fig. 15. The distribution of real SAC bugs found by DSAC.

be the sole correct referenced function of the function-pointer call. Thus, the detected bug about msleep on line 421 called by qla8044_fw_dump through qla8044_idc_lock is a false positive.

Secondly, our path-check method fails to identify infeasible code paths in some complex cases. The case of acquiring a spinlock under a condition and checking it across function calls is an example, and Figure 16(b) shows a false reported bug in such case. The function adpt_abort acquires a spinlock on line 705 under the condition that pHba->host is true on line 704, and then calls the function adpt_i2o_post_wait on line 706. The function adpt_i2o_post_wait releases the spinlock on line 1231 under a condition that pHba->host is true on line 1230, and then calls a sleep-able function schedule on line 1233. Thus, schedule is not called while holding the spinlock. However, DSAC does not maintain the condition pHba->host on line 704 across function calls, because this variable is a data-structure field, which the path-check method does not handle at present. Thus, our path-check method cannot use this condition to validate the path feasibility, and considers that schedule can be called while holding the spinlock. Thus, it reports a false bug.

\subsection{False Negatives}

To analyze the false negatives of DSAC, we compare its bug reports to the kernel commits fixing SAC bugs by changing GFP_KERNEL into GFP_ATOMIC between Linux 4.17 and 5.2 (June 2018 - July 2019). Specifically, we collect the commits that were not submitted by us and where the bug was already present in Linux v4.17. This strategy resulted in 16 commits. DSAC finds the bugs in 5 of these commits, but misses the bugs in the remaining 11 commits. These bugs are missed for two main reasons: 


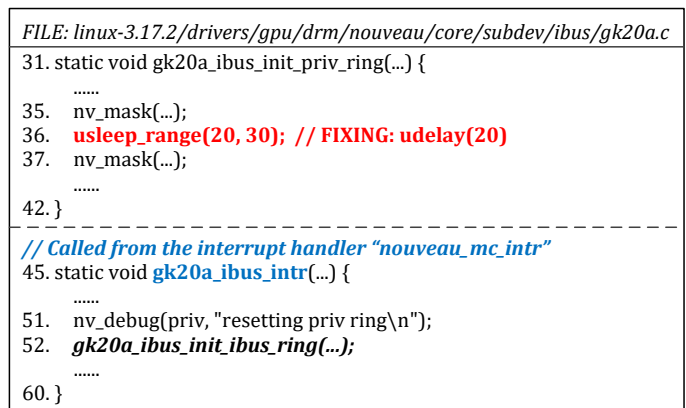

(a) P1

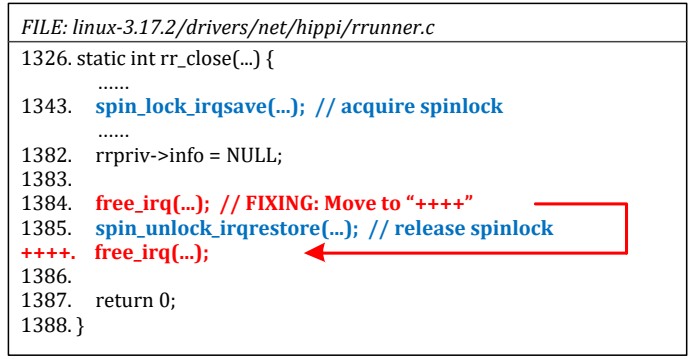

(c) P3

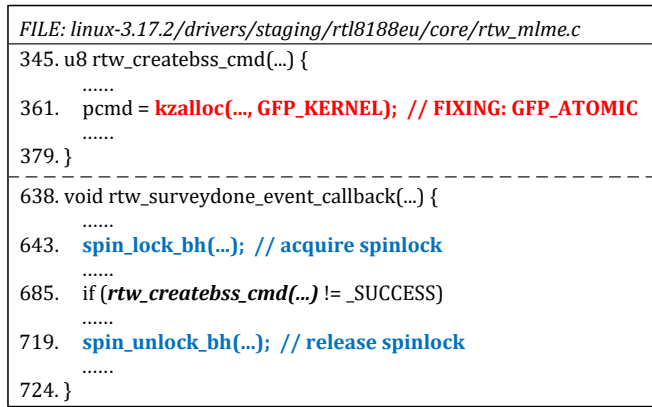

\section{(b) P2}

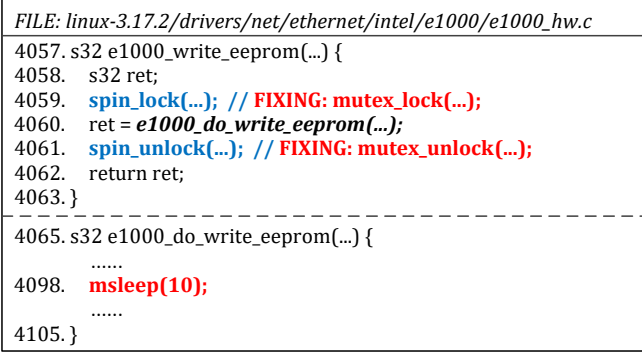

(d) P4

Fig. 17. Examples of common fixing patterns for SAC bugs in Linux 3.17.2.

P2: Replace the specific sleep-able constant flag with a non-sleep flag, as illustrated by the change GFP_KERNEL $\Rightarrow$ GFP_ATOMIC shown in Figure $17(\mathrm{~b}){ }^{6}$

P3: Move the sleep-able function call to some place where a spinlock is not held, as illustrated by the change of moving free_irq shown in Figure 17(c). ${ }^{7}$

P4: Replace the spinlock with a lock that allows sleeping, as illustrated by the change of spin_lock $\Rightarrow$ mutex_lock and spin_unlock $\Rightarrow$ mutex_unlock shown in Figure $17(\mathrm{~d}) .{ }^{8}$

These patterns have different usage scenarios and raise different challenges. Firstly, P1 and P2 can be used for all atomic contexts, while P3 and P4 are only used when holding a spinlock. Secondly, P1 and P2 involve simple modifications, while P3 and P4 involve more difficult modifications and are error-prone. Using P3 requires carefully determining where the sleep-able function should be moved to. Using P4 requires modifying all locking and unlocking operations. Thus, when we write our patches for fixing the detected bugs in Linux 4.17, we preferentially select the bugs that can be fixed using P1 and P2.

\subsection{Sensitivity Analysis}

DSAC exploits two key techniques: a summary-based analysis to reduce repeated analysis, and a connection-based alias analysis to identify the set of functions referenced by a function pointer. To better understand the value of these two techniques, we modify DSAC to drop each of them, and evaluate each modified tool on Linux 3.17.2.

Summary-based analysis. We implement a modified tool by dropping the summary-based analysis, implying that functions may be repeatedly analyzed in the same execution context. The

\footnotetext{
${ }^{6}$ Patch link: https://lkml.org/lkml/2017/12/18/833

${ }^{7}$ Patch link: https://patchwork.ozlabs.org/patch/847407/

${ }^{8}$ Patch link: https://patchwork.ozlabs.org/patch/499802/
} 
PathHasExisted check is retained, however, on lines 1-3 of HandleBlock (Figure 3), to protect against infinite loops due to recursion. In this experiment, the modified tool runs for over 11 hours and finally aborts due to insufficient memory, without completing the analysis. This experiment indicates that our summary-based analysis indeed improves the efficiency of atomic context analysis.

Connection-based alias analysis. We implement a modified tool by dropping the connectionbased alias analysis, and simply using a field-based analysis [30]. Specifically, we simply select all possible referenced functions of a function pointer stored in a data-structure field, according to its data-structure type, field offset and function-pointer type. In the experiment, the modified tool runs for around 320 minutes, and identifies more than $136 \mathrm{~K}$ referenced functions of function-pointer calls, which is much more than the number of referenced functions $(40 \mathrm{~K})$ identified by original DSAC. Because these referenced functions have to be all handled in the code analysis, the time usage of the modified tool is much longer than that of original DSAC (320 minutes vs. 78 minutes, i.e., over $4 \mathrm{x}$ longer). As for bug detection, the modified tool reports $2958 \mathrm{SAC}$ bugs, much more than the number of bugs found by original DSAC. We have manually checked these reported bugs, and found that more than 2000 of them are false. The reason is that in the recorded code paths of these false bugs, the referenced functions of function-pointer calls are incorrect. This experiment indicates that our connection-based alias analysis indeed reduces the false positive rate of SAC bug detection and improves the efficiency of atomic context analysis.

\section{COMPARISON TO PREVIOUS APPROACHES}

Several previous approaches $[4,7,15,24,46]$ have considered SAC bugs. Among them, we select two state-of-the-art approaches, namely the Coccinelle BlockLock checker [46] and our previous DSAC approach [7] to make detailed comparisons. We select the Coccinelle BlockLock checker because it has been used to detect many SAC bugs in the Linux kernel and it is open-source and its bug reports are available [11]. We select the previous DSAC approach because we aim to show the improvements of the current DSAC approach in this paper.

\subsection{Coccinelle BlockLock Checker}

Compared to the BlockLock checker, DSAC has some important improvements:

Atomic context analysis. BlockLock only uses one bit of context information to check if a lock is held, so it may not accurately identify the code in atomic context when multiple locks are taken but only some of them are released. DSAC maintains a complete lock stack during its summary-based analysis, thus it can be more accurate in identifying the code in atomic context. BlockLock is also not sensitive to the module Makefile, and thus may choose the wrong definition when unfolding a function call if the called function has multiple definitions. DSAC uses the module Makefile to accurately identify the definition of each function. DSAC can detect SAC bugs in interrupt handlers and involving sleep-able operations other than a call to an allocation function with GFP_KERNEL, which are not considered by BlockLock.

Function-pointer analysis. BlockLock does not handle function-pointer calls. DSAC uses a connection-based alias analysis to handle function-pointer calls. Thus, DSAC can find the bugs involving function-pointer calls, which are missed by BlockLock.

False bug filtering. BlockLock does not consider variable value information to validate path conditions, which may cause a number of false positives. DSAC uses a path-check method to validate whether the code path is feasible, which filters out many false bugs.

We also compare the experimental results of BlockLock and DSAC, with two steps. Firstly, we download the bug reports of BlockLock for Linux 2.6.33, and get 70 reported bugs. We select the bugs related to the $\mathrm{x} 86$ architecture based on the Kconfig files, leaving 37 reported SAC bugs (26 real bugs and 11 false bugs). Secondly, we use DSAC to check the source code of Linux 2.6.33. We 
use the kernel configuration allyesconfig to enable all code for the $\mathrm{x} 86$ architecture. DSAC runs for $45 \mathrm{~m}$ on four running threads, and finds 772 SAC bugs. We manually check these bugs, and find that 719 are real.

By manually comparing the bug reports, we find that: (1) 59 real bugs reported by DSAC are equivalent to 26 real bugs reported by BlockLock. DSAC reports more bugs because it detects basic sleep-able kernel interfaces, while BlockLock detects sleep-able functions. Thus, if a function defined in the kernel module calls several basic sleep-able kernel interfaces in atomic context, DSAC reports all these kernel interfaces, while BlockLock only reports this function. (2) DSAC filters out all false bugs reported by BlockLock. (3) DSAC reports 660 real bugs missed by BlockLock. Some of these bugs involve multiple source files, and BlockLock cannot handle them very precisely; some of these bugs involve function-pointer calls or are related to interrupt handling, which are not considered by BlockLock. (4) The false positive rate of DSAC is $6.9 \%$, which is lower than that of BlockLock.

However, compared to BlockLock, an important limitation of DSAC is that its results are specific to a single kernel configuration. BlockLock is based on Coccinelle [45], which does not compile the source code. Thus it can conveniently check all source files without any kernel configuration. DSAC is based on LLVM, which compiles the source code with a selected kernel configuration. Thus, the 33 bugs found by BlockLock for non-x 86 architectures are missed by DSAC.

\subsection{Our Previous DSAC approach}

Compared to our previous DSAC approach [7], our current DSAC approach achieves some important improvements:

Summary-based analysis. Our previous DSAC approach uses a hybrid of flow-sensitive and -insensitive analysis, which may repeatedly analyze a function under the same execution context. Our current DSAC approach uses a summary-based analysis, which only analyzes a function once per execution context. And our current DSAC approach uses a flow-sensitive analysis to analyze all targeted code paths. Thus, our current DSAC approach is more efficient and accurate than our previous DSAC approach.

Cross-kernel-module analysis. Our previous DSAC approach handles one kernel module at a time and only analyzes code paths within the module. Our current DSAC approach can handle multiple kernel modules and can analyze code paths across different kernel modules. Thus, our current DSAC approach can find bugs involving multiple kernel modules that are missed by our previous DSAC approach.

Connection-based alias analysis. Our previous DSAC approach does not handle functionpointer calls. Our current DSAC approach uses a connection-based alias analysis to identify the set of functions referenced by a function pointer. Thus, our current DSAC approach can find many bugs involving function-pointer calls that are missed by our previous DSAC approach.

Improved path-check method. Our previous DSAC approach relied on some information about common coding styles to check the names of variables and called functions in if conditions. This approach is only applicable to the cases that follow the expected coding styles, and thus many false bugs involving other cases are still reported. Our current DSAC approach collects some information about variable values to validate the feasibility of the code path, which is more accurate and effective in filtering out false bugs.

Our previous DSAC and current DSAC approaches are both evaluated on Linux 3.17.2. However, our previous DSAC approach only checks device drivers, while our current DSAC approach checks the whole kernel. To make a fair comparison, we select the SAC bugs of drivers found by our current DSAC approach, and get 666 bug reports (627 real bugs and 39 false bugs). Our previous DSAC approach reports 215 bugs in drivers (200 real bugs and 15 false bugs). By manually checking 
the bug reports, we find that: (1) The 200 real bugs found by our previous DSAC approach are all found by our current DSAC approach. (2) Among the 15 false bugs found by our previous DSAC approach, 10 are filtered out by our current DSAC approach. (3) Our current DSAC approach finds 427 real bugs missed by our previous DSAC approach. These bugs involve multiple kernel modules or function-pointer calls, and our previous DSAC approach cannot handle them. (4) The false positive rate of our current DSAC approach is $5.9 \%$, which is lower than that of our previous DSAC approach (7.0\%).

\section{DISCUSSION}

In this section, we discuss two possible extensions for DSAC.

\subsection{Detecting SAC Bugs in Other OS Kernels}

At present, DSAC is specific to the Linux kernel, in two main aspects. Firstly, DSAC requires LLVM bytecode and link information for the OS kernel, and we implement a script specific to the Linux kernel to collect this information when compiling the Linux kernel source code. Secondly, DSAC requires the names of some functions and flags specific to the Linux kernel. For the summary-based analysis in Figure 3, we need to manually provide the names of the spin-lock and spin-unlock functions used on lines 6 and 8 in HandleBlock and on line 3 in CodeAnalysis, the names of the interrupt-handler-register functions used on line 8 in CodeAnalysis, and the names of the basic sleep-able kernel interfaces and sleep-able flags used on lines 10-11 in CodeAnalysis. For the pathcheck method, we need to manually provide the names of the functions checking atomic context and the non-sleep flags. Thus, to apply DSAC in another OS kernel, such as FreeBSD or NetBSD, we need to implement a new script for the source-code compilation of this kernel, and provide the names of the corresponding functions and flags specific to the targeted kernel.

\subsection{Detecting Other Kernel Problems}

The good results and reasonable performance of DSAC rely on three key techniques used in DSAC: function summaries to avoid repeated analysis, link and file connections to reduce the set of considered functions when analyzing function-pointer calls, and a path-check method that only applies precise analysis to possible bug reports to avoid the cost of always checking during static analysis. DSAC also benefits from the fact that it is looking for a specific bug type, involving specific operators, such as locking calls and sleep-able functions, that occur frequently, but not pervasively. Other problems that may have similar properties include properties of specific resource allocations, such as double locks or memory leaks. We are currently developing a general interprocedural program analysis framework targeting the Linux kernel in order to be apply the techniques used by DSAC to a wider range of bug types.

\section{RELATED WORK}

\subsection{Detecting Concurrency Bugs}

Many approaches $[13,21,26,35,37,44,50,55,56]$ have been proposed to detect concurrency bugs in user-mode applications. Some of them $[13,26,55]$ use dynamic analysis to collect and analyze runtime information to detect concurrency bugs. But the code coverage of dynamic analysis is limited by test cases. Others [21, 44, 50,56] use static analysis to cover more code without running the tested programs. But static analysis often introduces false positives. Some approaches [14, 35, 37] combine static and dynamic analysis to achieve higher code coverage with fewer false positives DSAC uses static analysis to cover the whole kernel, and validates the feasibility of code paths to reduce false positives. 
To improve OS reliability, some approaches [20, 23, 25, 27, 53, 54, 57] detect some kinds of concurrency bugs like data races, but they do not detect SAC bugs. Several approaches $[4,15,24,46]$ can detect common kinds of OS kernel bugs, including SAC bugs. But they are not specific to SAC bugs, and most of them $[15,24,46]$ are designed to collect statistics rather than report specific bugs to the user, making issues such as detection time and false positive rate less important. Besides, these approaches does not handle function pointers, so they may miss SAC bugs that involve function-pointer calls. For example, BlockLock [46] has an overall false positive rate of $20 \%$, while that of DSAC is less than $10 \%$, and BlockLock also misses many real bugs found by DSAC, especially the bugs involving function-pointer calls.

\subsection{Checking API Rules}

Checking API rules is a promising way of finding deep and semantic bugs in an OS kernel. Some approaches $[6,10,40,42]$ use known API rules to statically or dynamically detect API misuses. For example, with known paired kernel reference count management interfaces, RID [40] uses a summary-based inter-procedural analysis to detect reference counting bugs in the Linux kernel. To find implicit API rules, some approaches do specification mining by analyzing source code [33, $36,38,49,59]$ or execution traces [8, 31, 58], and then use the mined API rules to detect violations. For example, PF-Miner [38] analyzes the source code of the Android kernel. It first identifies error handling paths from $\mathrm{C}$ source code, and then respectively collects function call sequences in normal execution paths and error handling paths. By using statistical methods to compare the collected function call sequences in the two kinds of code paths, PF-Miner extracts frequently used function pairs as API rules. Perracotta [58] analyzes execution traces for the Windows kernel. It partitions the original imperfect execution traces into some sub-traces, and identifies different objects using context-sensitive analysis. Then, Perracotta uses three heuristics, namely call-graph reachability, name similarity and properties combination, to find interesting API rules.

Most of these approaches focus on the temporal rules of common API usages, such as resource acquiring and releasing pairs $[49,58]$ and error handling patterns $[8,33,38]$, but these approaches have not targeted SAC bugs.

\subsection{Function-Pointer Analysis}

In static analysis, analyzing function pointers is a classical and difficult problem, because the set of functions referenced by a function pointer is often hard to correctly identify without exact runtime information. Some alias-analysis approaches [22, 29, 30, 32, 41] have been proposed that can handle function pointers. They are classified as Andersen-style [3] or Steensgaard-style [51]. Andersen-style approaches view pointer assignments as subset constraints, and use constraints to propagate pointer information; Steensgaard-style approaches also use constraint-based analysis, but they use equality constraints instead of subset constraints. CLA [30] uses a field-based and flowinsensitive pointer analysis to analyze large-scale software in a short time. It is Andersen-style, and uses the data structure type and field name to maintain field sensitivity. DSA [32] is field-sensitive, context-sensitive and flow-insensitive pointer analysis algorithm. DSA is Steensgaard-style [51] with full heap cloning, and uses some practical optimizations to speed up alias analysis.

However, these approaches often identify incorrect referenced functions of function pointers in large and complex software. A main reason is that they do not consider the calling context of a function-pointer call, and just produce all the functions possibly referenced by the called function pointer. In this paper, based on the field-based analysis of CLA [30], we propose connection-based alias analysis to improve the accuracy of analyzing function-pointer calls. 


\subsection{Improving the Kernel Module Architecture}

To prevent concurrency bugs in the OS kernel, several improved kernel module architectures have been proposed, typically for device drivers. The active driver architecture $[2,48]$ runs each driver in a separate kernel thread, and all communication between the driver and kernel is performed using message passing. This architecture can serialize the concurrent accesses to the driver and eliminate the possibility of concurrency bugs. The user-mode driver architecture [28, 34, 47] runs each driver in a separate user-mode process. This architecture protects the OS kernel against crashes caused by driver code. This architecture also allows the driver code to be implemented using a safer language such as Java [47], instead of C.

These approaches have two main limitations. Firstly, the source code of the kernel module must be manually rewritten. Secondly, the performance of the kernel module may degrade due to serialization in the active driver approaches and frequent context switches in the user-mode driver approaches.

\section{CONCLUSION}

In this paper, we have proposed DSAC, a practical static approach, to automatically and effectively detect SAC bugs in the Linux kernel. DSAC uses three key techniques: (1) a summary-based analysis to identify the code that may be executed in atomic context. (2) a connection-based alias analysis to identify the set of functions referenced by a function pointer. (3) a path-check method to filter out repeated reports and false bugs. We have used DSAC to check the kernel source code of Linux 4.17, and in total find 1068 real bugs. We have randomly selected 300 of the real bugs and sent them to kernel developers. 220 of these bugs have been confirmed, and 51 of our patches fixing 115 bugs have been applied.

DSAC can be still improved in several aspects. Firstly, the current implementation of DSAC can be improved to reduce false positives. For example, DSAC still fails to correctly validate the feasibility of the code path in complex cases. Thus, we need to improve our path-check method to accurately handle such cases. Secondly, at present, DSAC focuses on function pointers stored in data-structure fields, and cannot handle function pointers that are not referenced in this way. Thus, we need to improve our connection-based alias analysis to handle more kinds of function-pointer calls, which can help to find more SAC bugs. Thirdly, we have only evaluated DSAC on the Linux kernel in this paper. In fact, our previous DSAC approach [7] also found some real SAC bugs in the FreeBSD and NetBSD kernels. Thus, we will evaluate DSAC on these OS kernels. Finally, our connection-based alias analysis can help to build a full kernel call graph involving function-pointer calls. We are investigating whether the collected information is sufficient to accurately support detection of other kinds of kernel problems, such as double free and double lock bugs.

\section{ACKNOWLEDGMENT}

We would like to thank the Linux kernel developers and maintainers who gave helpful feedback on our bug reports and patches. This work was supported in part by the China Postdoctoral Science Foundation under Project 2019T120093. Shi-Min Hu is the corresponding author.

\section{REFERENCES}

[1] Allocation 2018. Linux kernel documentation for memory allocation. https://www.kernel.org/doc/htmldocs/kernelapi/API-kmalloc.html.

[2] Sidney Amani, Peter Chubb, Alastair F Donaldson, Alexander Legg, Keng Chai Ong, Leonid Ryzhyk, and Yanjin Zhu. 2014. Automatic verification of active device drivers. ACM SIGOPS Operating Systems Review 48, 1 (2014), 106-118.

[3] Lars Ole Andersen. 1994. Program analysis and specialization for the C programming language. Ph.D. Dissertation. University of Cophenhagen. 
[4] Zachary R Anderson, Eric A Brewer, Jeremy Condit, Robert Ennals, David Gay, Matthew Harren, George C Necula, and Feng Zhou. 2007. Beyond bug-finding: sound program analysis for Linux. In Proceedings of the 11th International Workshop on Hot Topics in Operating Systems (HotOS). 1-6.

[5] Jia-Ju Bai, Julia Lawall, Wende Tan, and Shi-Min Hu. 2019. DCNS: automated detection of conservative non-sleep defects in the Linux kernel. In Proceedings of the 24th International Conference on Architectural Support for Programming Languages and Operating Systems (ASPLOS). 287-299.

[6] Jia-Ju Bai, Hu-Qiu Liu, Yu-Ping Wang Wang, and Hu Shi-Min. 2014. aComment: mining annotations from comments and code to detect interrupt related concurrency bugs. In Proceedings of the 21st Asia-Pacific Software Engineering Conference (APSEC). 407-414.

[7] Jia-Ju Bai, Yu-Ping Wang, Julia Lawall, and Shi-Min Hu. 2018. DSAC: effective static analysis of sleep-in-atomic-context bugs in kernel modules. In Proceedings of the 2018 USENIX ATC Conference (USENIX ATC). 587-600.

[8] Jia-Ju Bai, Yu-Ping Wang, Hu-Qiu Liu, and Shi-Min Hu. 2016. Mining and checking paired functions in device drivers using characteristic fault injection. Information and Software Technology 73 (2016), 122-133.

[9] Arati Baliga, Vinod Ganapathy, and Liviu Iftode. 2011. Detecting kernel-level rootkits using data structure invariants. IEEE Transactions on Dependable and Secure Computing (TDSC) 8, 5 (2011), 670-684.

[10] Thomas Ball, Ella Bounimova, Byron Cook, Vladimir Levin, Jakob Lichtenberg, Con McGarvey, Bohus Ondrusek, Sriram K Rajamani, and Abdullah Ustuner. 2006. Thorough static analysis of device drivers. In Proceedings of the 1st European Conference on Computer Systems (EuroSys). 73-85.

[11] BlockLock 2014. Website for "Faults in Linux: ten years later". http://faultlinux.lip6.fr/.

[12] Cristian Cadar, Daniel Dunbar, and Dawson Engler. 2008. KLEE: unassisted and automatic generation of high-coverage tests for complex systems programs. In Proceedings of the 8th International Conference on Operating Systems Design and Implementation (OSDI). 209-224.

[13] Yan Cai, Jian Zhang, Lingwei Cao, and Jian Liu. 2016. A deployable sampling strategy for data race detection. In Proceedings of the 24th International Symposium on Foundations of Software Engineering (FSE). 810-821.

[14] Lee Chew and David Lie. 2010. Kivati: fast detection and prevention of atomicity violations. In Proceedings of 5th European Conference on Computer Systems (EuroSys). 307-320.

[15] Andy Chou, Junfeng Yang, Benjamin Chelf, Seth Hallem, and Dawson Engler. 2001. An empirical study of operating systems errors. In Proceedings of the 18th International Symposium on Operating Systems Principles (SOSP). 73-88.

[16] Clang 2018. Clang compiler. http://clang.llvm.org/.

[17] CLOC 2018. CLOC: counting lines of code. https://github.com/AlDanial/cloc.

[18] Jonathan Corbet. 2008. Atomic context and kernel API design. https://wn.net/Articles/274695/.

[19] Domenico Cotroneo, Roberto Natella, and Stefano Russo. 2009. Assessment and improvement of hang detection in the Linux operating system. In Proceedings of the 28th International Symposium on Reliable Distributed Systems (SRDS). 288-294.

[20] Pantazis Deligiannis, Alastair F Donaldson, and Zvonimir Rakamaric. 2015. Fast and precise symbolic analysis of concurrency bugs in device drivers. In Proceedings of the 30th International Conference on Automated Software Engineering (ASE). 166-177.

[21] Jyotirmoy Deshmukh, E Allen Emerson, and Sriram Sankaranarayanan. 2009. Symbolic deadlock analysis in concurrent libraries and their clients. In Proceedings of the 24th International Conference on Automated Software Engineering (ASE). 480-491.

[22] Maryam Emami, Rakesh Ghiya, and Laurie J Hendren. 1994. Context-sensitive interprocedural points-to analysis in the presence of function pointers. In Proceedings of the 1994 International Conference on Programming Language Design and Implementation (PLDI). 242-256.

[23] Dawson Engler and Ken Ashcraft. 2003. RacerX: effective, static detection of race conditions and deadlocks. In Proceedings of the 19th International Symposium on Operating Systems Principles (SOSP). 237-252.

[24] Dawson Engler, Benjamin Chelf, Andy Chou, and Seth Hallem. 2000. Checking system rules using system-specific, programmer-written compiler extensions. In Proceedings of the 4th International Conference on Operating Systems Design and Implementation (OSDI). 1-16.

[25] John Erickson, Madanlal Musuvathi, Sebastian Burckhardt, and Kirk Olynyk. 2010. Effective data-race detection for the kernel. In Proceedings of the 9th International Conference on Operating Systems Design and Implementation (OSDI). 151-162.

[26] Pedro Fonseca, Cheng Li, and Rodrigo Rodrigues. 2011. Finding complex concurrency bugs in large multi-threaded applications. In Proceedings of the 6th European Conference on Computer Systems (EuroSys). 215-228.

[27] Pedro Fonseca, Rodrigo Rodrigues, and Björn B Brandenburg. 2014. SKI: exposing kernel concurrency bugs through systematic schedule exploration. In Proceedings of the 11th International Conference on Operating Systems Design and Implementation (OSDI). 415-431. 
[28] Vinod Ganapathy, Matthew J Renzelmann, Arini Balakrishnan, Michael M Swift, and Somesh Jha. 2008. The design and implementation of microdrivers. In Proceedings of the 13th International Conference on Architectural Support for Programming Languages and Operating Systems (ASPLOS). 168-178.

[29] Ben Hardekopf and Calvin Lin. 2011. Flow-sensitive pointer analysis for millions of lines of code. In Proceedings of the 9th International Symposium on Code Generation and Optimization (CGO). 289-298.

[30] Nevin Heintze and Olivier Tardieu. 2001. Ultra-fast aliasing analysis using CLA: a million lines of C code in a second. In Proceedings of the 2001 International Conference on Programming Language Design and Implementation (PLDI). 254-263.

[31] Christopher LaRosa, Li Xiong, and Ken Mandelberg. 2008. Frequent pattern mining for kernel trace data. In Proceedings of the 2008 ACM symposium on Applied computing. 880-885.

[32] Chris Lattner, Andrew Lenharth, and Vikram Adve. 2007. Making context-sensitive points-to analysis with heap cloning practical for the real world. In Proceedings of the 2007 International Conference on Programming Language Design and Implementation (PLDI). 278-289.

[33] Julia L Lawall, Julien Brunel, Nicolas Palix, René Rydhof Hansen, Henrik Stuart, and Gilles Muller. 2009. WYSIWIB: A declarative approach to finding API protocols and bugs in Linux code. In Proceedings of the 39th International Conference on Dependable Systems and Networks (DSN). 43-52.

[34] Ben Leslie, Peter Chubb, Nicholas Fitzroy-Dale, Stefan Götz, Charles Gray, Luke Macpherson, Daniel Potts, Yue-Ting Shen, Kevin Elphinstone, and Gernot Heiser. 2005. User-level device drivers: achieved performance. Fournal of Computer Science and Technology 20, 5 (2005), 654-664.

[35] Qiwei Li, Yanyan Jiang, Tianxiao Gu, Chang Xu, Jun Ma, Xiaoxing Ma, and Jian Lu. 2016. Effectively manifesting concurrency bugs in Android apps. In Proceedings of the 23rd Asia-Pacific Software Engineering Conference (APSEC) 209-216.

[36] Zhenmin Li and Yuanyuan Zhou. 2005. PR-Miner: automatically extracting implicit programming rules and detecting violations in large software code. In Proceedings of the 13th International Symposium on Foundations of Software Engineering (FSE). 306-315.

[37] Haopeng Liu, Guangpu Li, Jeffrey F Lukman, Jiaxin Li, Shan Lu, Haryadi S Gunawi, and Chen Tian. 2017. DCatch: automatically detecting distributed concurrency bugs in cloud systems. In Proceedings of the 22nd International Conference on Architectural Support for Programming Languages and Operating Systems (ASPLOS). 677-691.

[38] Hu-Qiu Liu, Yu-Ping Wang, Jia-Ju Bai, and Shi-Min Hu. 2016. PF-Miner: a practical paired functions mining method for Android kernel in error paths. Journal of Systems and Software 121 (2016), 234-246.

[39] LLVM 2018. LLVM compiler infrastructure. https://lvm.org/.

[40] Junjie Mao, Yu Chen, Qixue Xiao, and Yuanchun Shi. 2016. RID: finding reference count bugs with inconsistent path pair checking. In Proceedings of the 21st International Conference on Architectural Support for Programming Languages and Operating Systems (ASPLOS). 531-544.

[41] Ana Milanova, Atanas Rountev, and Barbara G Ryder. 2004. Precise call graphs for C programs with function pointers. Automated Software Engineering 11, 1 (2004), 7-26.

[42] Changwoo Min, Sanidhya Kashyap, Byoungyoung Lee, Chengyu Song, and Taesoo Kim. 2015. Cross-checking semantic correctness: the case of finding file system bugs. In Proceedings of the 25th Symposium on Operating Systems Principles (SOSP). 361-377.

[43] MySQL 2018. MYSQL database. https://www.mysql.com/.

[44] Mayur Naik, Alex Aiken, and John Whaley. 2006. Effective static race detection for Java. In Proceedings of the 27th International Conference on Programming Language Design and Implementation (PLDI). 308-319.

[45] Yoann Padioleau, Julia Lawall, René Rydhof Hansen, and Gilles Muller. 2008. Documenting and automating collateral evolutions in Linux device drivers. In Proceedings of the 3rd European Conference on Computer Systems (EuroSys) 247-260

[46] Nicolas Palix, Gaēl Thomas, Suman Saha, Christophe Calvès, Gilles Muller, and Julia Lawall. 2014. Faults in Linux 2.6 ACM Transactions on Computer Systems (TOCS) 32, 2 (2014), 4:1-4:40.

[47] Matthew J Renzelmann and Michael M Swift. 2009. Decaf: Moving device drivers to a modern language. In USENIX Annual Technical Conference (USENIX ATC). 1-14.

[48] Leonid Ryzhyk, Yanjin Zhu, and Gernot Heiser. 2010. The case for active device drivers. In Proceedings of the 1st Aisa-Pacific Workshop on Systems (APSys). 25-30.

[49] Suman Saha, Jean-Pierre Lozi, Gaël Thomas, Julia L Lawall, and Gilles Muller. 2013. Hector: Detecting resource-release omission faults in error-handling code for systems software. In Proceedings of the 43rd International Conference on Dependable Systems and Networks (DSN). 1-12.

[50] Anirudh Santhiar and Aditya Kanade. 2017. Static deadlock detection for asynchronous C\# programs. In Proceedings of the 38th International Conference on Programming Language Design and Implementation (PLDI). 292-305.

[51] Bjarne Steensgaard. 1996. Points-to analysis in almost linear time. In Proceedings of the 23rd International Symposium on Principles of Programming Languages (POPL). 32-41. 
[52] Michael M. Swift, Brian N. Bershad, and Henry M. Levy. 2003. Improving the reliability of commodity operating systems. In Proceedings of the 19th International Symposium on Operating Systems Principles (SOSP). 207-222.

[53] Lin Tan, Yuanyuan Zhou, and Yoann Padioleau. 2011. aComment: mining annotations from comments and code to detect interrupt related concurrency bugs. In Proceedings of the 33rd International Conference on Software Engineering (ICSE). 11-20.

[54] Vesal Vojdani, Kalmer Apinis, Vootele Rõtov, Helmut Seidl, Varmo Vene, and Ralf Vogler. 2016. Static race detection for device drivers: the Goblint approach. In Proceedings of the 31st International Conference on Automated Software Engineering (ASE). 391-402.

[55] Dasarath Weeratunge, Xiangyu Zhang, William N Sumner, and Suresh Jagannathan. 2010. Analyzing concurrency bugs using dual slicing. In Proceedings of the 19th International Symposium on Software Testing and Analysis (ISSTA) 253-264.

[56] Amy Williams, William Thies, and Michael D Ernst. 2005. Static deadlock detection for Java libraries. In Proceedings of the 19th European Conference on Object-Oriented Programming (ECOOP). 602-629.

[57] Thomas Witkowski, Nicolas Blanc, Daniel Kroening, and Georg Weissenbacher. 2007. Model checking concurrent linux device drivers. In Proceedings of the 22nd International Conference on Automated Software Engineering (ASE). 501-504.

[58] Jinlin Yang, David Evans, Deepali Bhardwaj, Thirumalesh Bhat, and Manuvir Das. 2006. Perracotta: mining temporal API rules from imperfect traces. In Proceedings of 28th International Conference on Software Engineering (ICSE). 282-291.

[59] Insu Yun, Changwoo Min, Xujie Si, Yeongjin Jang, Taesoo Kim, and Mayur Naik. 2016. APISan: sanitizing API usages through semantic cross-checking. In USENIX Security Symposium. 363-378.

[60] Yian Zhu, Yue Li, Jingling Xue, Tian Tan, Jialong Shi, Yang Shen, and Chunyan Ma. 2012. What is system hang and how to handle it. In Proceedings of the 23rd International Symposium on Software Reliability Engineering (ISSRE). 141-150.

Received October 2018; revised September 2019; accepted xxx 